\title{
A Cahn-Hilliard-type equation with application to tumor growth dynamics
}

\author{
Abramo Agosti | Paola Francesca Antonietti | Pasquale Ciarletta | Maurizio Grasselli | \\ Marco Verani
}

We consider a Cahn-Hilliard-type equation with degenerate mobility and single-well potential of Lennard-Jones type. This equation models the evolution and growth of biological cells such as solid tumors. The degeneracy set of the mobility and the singularity set of the cellular potential do not coincide, and the absence of cells is an unstable equilibrium configuration of the potential. This feature introduces a nontrivial difference with respect to the Cahn-Hilliard equation analyzed in the literature. We give existence results for different classes Dipartimento di Matematica, Politecnico di Milano, via Bonardi 9, 20133 Milano, Italy

\section{Correspondence}

Abramo Agosti, Dipartimento di

Matematica, Politecnico di Milano, via

Bonardi 9, 20133 Milano, Italy.

Email: abramo.agosti@polimi.it of weak solutions. Moreover, we formulate a continuous finite element approximation of the problem, where the positivity of the solution is enforced through a discrete variational inequality. We prove the existence and uniqueness of the discrete solution for any spatial dimension together with the convergence to the weak solution for spatial dimension $d=1$. We present simulation results in 1 and 2 space dimensions. We also study the dynamics of the spinodal decomposition and the growth and scaling laws of phase ordering dynamics. In this case, we find similar results to the ones obtained in standard phase ordering dynamics and we highlight the fact that the asymptotic behavior of the solution is dominated by the mechanism of growth by bulk diffusion.

\section{KEYWORDS}

continuous Galerkin finite element approximation, degenerate Cahn-Hilliard equation, single well potential, tumor growth models

\section{1 | INTRODUCTION}

The Cahn-Hilliard (CH) equation has originally been proposed as a phenomenological model for phase separation in binary solutions due to cooling processes in Cahn and Hilliard ${ }^{1}$ and Cahn. ${ }^{2}$ In this phenomenon, the 2 phases spontaneously separate to form domains where pure components dominate (see, for instance, Lee et al, ${ }^{3}$ Novick-Cohen, ${ }^{4}$ and their references). The $\mathrm{CH}$ equation can be written as a mass continuity equation with a flux $J$, namely,

$$
\frac{\partial c}{\partial t}+\operatorname{div} J=0
$$

where $c \in[0,1]$ is the (relative) concentration of one component of the binary solution and $J$ is given by

$$
J=-b(c) \nabla \frac{\delta F}{\delta c}
$$

This is the peer reviewed version of the following article: "A Cahn-Hilliard-type equation with application to tumor growth dynamics", which has been published in final form at http://dx.doi.org/10.1002\%2Fmma.4548. This article may be used for non-commercial purposes in accordance with Wiley Terms and Conditions for Use of Self-Archived Versions 
where $b(c)$ is the mobility and $F$ is the total Helmholtz free energy defined by

$$
F(c)=\int_{\Omega}\left(\frac{\gamma}{2}|\nabla c|^{2}+\psi(c)\right) d x .
$$

Here, $\gamma$ is a positive material parameter related to the thickness of the diffuse interface separating the 2 phases and $\psi(c)$ is the homogeneous Helmholtz free energy density defined by

$$
\psi(c)=\frac{\theta}{2}(c \log (c)+(1-c) \log (1-c))-\frac{\theta_{*}}{2}\left(c-\frac{1}{2}\right)^{2},
$$

where $0<\theta<\theta *, \theta$ being the absolute temperature and $\theta *$ a given critical temperature below which the phase separation takes place. We recall that this logarithmic potential is often approximated by a smooth double-well potential defined on the whole real line.

Consequently, Equation 1 can be written as a fourth-order nonlinear evolution equation as follows:

$$
\frac{\partial c}{\partial t}=\operatorname{div}\left(b(c) \nabla\left(-\gamma \Delta c+\psi^{\prime}(c)\right)\right) .
$$

The theoretical aspects of Equation 3 have been investigated in many contributions, especially in the case where the mobility is a positive constant and $\psi$ is a smooth double-well potential. Regarding the latter, we just mention the pioneering results obtained in Elliott and Zheng, ${ }^{5}$ while, for the logarithmic potential, the reader is referred to the review paper of Cherfils et al. ${ }^{6}$ If the mobility is degenerate at the pure phases, namely, $b(c)=c(1-c)$, and $\psi$ is of logarithmic type, then a well-known result is the existence of a suitably weak solution, which has been established in Elliott and Garcke ${ }^{7}$ (see also Cahn et $\mathrm{al}^{8}$ for the interpretation of the sharp interface limit). Recently, in the case in which the singular character of the potential dominate over the degeneracy of the mobility, the existence of a weak solution in a suitable dual mixed formulation has been estabilished in Schimperna and Zelik. ${ }^{9}$

Cahn-Hilliard-type equations have been used in several different contexts. From the original one (ie, binary alloys) introduced in Allen and Cahn, ${ }^{10}$ to multicomponent polymeric systems in McMaster, ${ }^{11}$ and lithium-ion batteries in Zeng and Bazant, ${ }^{12}$ but also in modeling nanoporosity during dealloying in Erlebacher et al, ${ }^{13}$ or inpainting of binary images in Bertozzi et al, ${ }^{14}$ and even the formation of Saturn rings in Tremaine. ${ }^{15}$ Further generalizations include extensions to deformable elastic continua, as in Gurtin ${ }^{16}$ binary fluids (see, eg, Gurtin et $\mathrm{al}^{17}$ and Lowengrub and Truskinovsky ${ }^{18}$ ). In the last years, $\mathrm{CH}$-type equations have also been used to model pattern formation in biological systems (see, for instance, Khain and Sander ${ }^{19}$ and Liu et $\mathrm{al}^{20}$ ). Moreover, a $\mathrm{CH}$ equation with degenerate mobility obtained from the application of mixture theory to solid tumors, which are modeled as elastic fluids with a free energy functional characterized by a double-well potential $\psi$, is described in Wise et al. ${ }^{21}$

The standard initial and boundary value problem associated with (3) is the following:

Problem P. Find $c(\mathbf{x}, t)$ such that

$$
\begin{gathered}
\frac{\partial c}{\partial t}=\operatorname{div}\left(b(c) \nabla\left(-\gamma \Delta c+\psi^{\prime}(c)\right)\right) \quad \text { in } \Omega_{T}:=\Omega \times(0, T), \\
c(\mathbf{x}, 0)=c_{0}(\mathbf{x}) \quad \forall \mathbf{x} \in \Omega, \\
\nabla c \cdot v=b(c) \nabla\left(-\gamma \Delta c+\psi^{\prime}(c)\right) \cdot v=0 \quad \text { on } \partial \Omega \times(0, T),
\end{gathered}
$$

where $\Omega \subset \mathbb{R}^{d}, d=1,2,3$ is a given bounded domain with a Lipschitz boundary $\partial \Omega, v$ is the unit normal vector pointing outward to $\partial \Omega$ and $c_{0}$ is a given initial concentration.

A remarkable property of the solution of Problem $\mathrm{P}$ in the degenerate case with the smooth or the logarithmic double well potential is the positivity property, ie, if $0 \leqslant c_{0}(\mathbf{x}) \leqslant 1$, then $0 \leqslant c(\mathbf{x}, t) \leqslant 1$ almost everywhere in $\Omega_{T}$. See, for instance, other works ${ }^{7,922}$ for details. As we will see in the following, this property is not satisfied in general by the solution of a finite element approximation of Problem P. See, for instance, Barrett et al ${ }^{23}$ and Grün and Rumpf ${ }^{24}$ for details.

Finite element discretizations of Problem P using continuous elements have been studied first in Copetti and Elliott ${ }^{25}$ for a constant mobility and in Barrett et $\mathrm{al}^{23}$ and Grün and Rumpf $\mathrm{f}^{24}$ for degenerate mobility (for more recent contributions, see, eg, previous studies ${ }^{26,27}$ where an isogeometric analysis of the $\mathrm{CH}$ equation has been performed; 


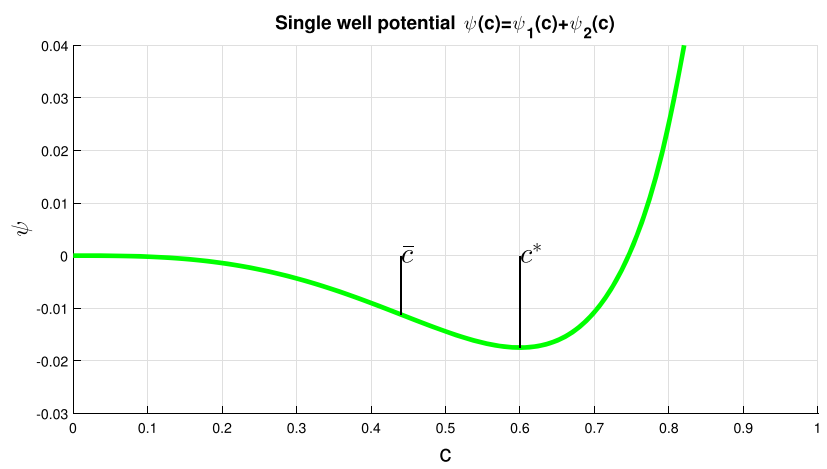

FIGURE 1 Plot of the single well potential (7) corresponding to the value $c^{*}=0.6$, with $\bar{c} \sim 0.44$ [Colour figure can be viewed at wileyonlinelibrary.com]

other studies ${ }^{28-30}$ where a finite element discretization with discontinuous elements has been studied, and another two studies, ${ }^{31,32}$ where polygonal methods are used). Whereas, in Barrett et al, ${ }^{23}$ the positivity property of the discrete solution is imposed as a constraint by means of a variational inequality, in Grün and Rumpf, ${ }^{24}$ a suitable approximation of the degenerate mobility by means of an harmonic average on a structured mesh is used, to guarantee the validity of a discrete positivity property.

In these papers, $\psi$ is always a double-well smooth or logarithmic type potential. However, in modeling tumor growth, such a choice seems unphysical for biological cells, since it has been observed that cell-cell interactions are attractive at a moderate cell volume fraction (for $c<c^{*}$ ), where $c^{*} \in \mathbb{R}, 0<c^{*}<1$, and repulsive at a high volume fraction (for $c>c^{*}$ ), with a zero for $c=0$ and an infinite cell-cell repulsion as $c \rightarrow 1$, (see, eg, Byrne and Preziosi ${ }^{33}$ ). Here, $c$ stands for the volume fraction of cancerous cells.

Accordingly, in this work, we propose to study the existence of a suitable weak solution and the convergence of a finite element approximation of Problem P, using a single-well potential of Lennard-Jones type, as introduced in Chatelain et $\mathrm{al}^{34}$ (cf also Colombo et $\mathrm{al}^{35}$ ). More precisely, we take

$$
\psi(c)=\psi_{1}(c)+\psi_{2}(c)
$$

where

$$
\begin{aligned}
& \psi_{1}(c)=-\left(1-c^{*}\right) \log (1-c), \\
& \psi_{2}(c)=-\frac{c^{3}}{3}-\left(1-c^{*}\right) \frac{c^{2}}{2}-\left(1-c^{*}\right) c+k .
\end{aligned}
$$

Here, $c^{*}$ is the volume fraction at which the cells would naturally be at mutual equilibrium and $k>0$. The spinodal decomposition can be triggered if $c<\bar{c}$, where $\psi^{\prime \prime}(\bar{c})=0$. In Figure 1, a plot of the single well potential (7), corresponding to the value $c^{*}=0.6$, is shown.

Moreover, we have

$$
\psi^{\prime}(c)=\frac{c^{2}\left(c-c^{*}\right)}{1-c}
$$

Correspondingly, the mobility introduced in Chatelain et $\mathrm{ll}^{36}$ is given by

$$
b(c)=c(1-c)^{2} .
$$

Note that $\psi_{1}$ is a convex function defined on $[0,1)$ while $\psi_{2}$ is a concave function defined on $[0,1]$. The particular form of the mobility (10) is derived in Chatelain et $\mathrm{al}^{36}$ as a result of the application of mixture model to solid tumors. We observe in particular that the product $b \psi^{\prime \prime}$ is a continuous function in $[0,1]$. We remark the fact that the analysis of Problem P could be extended easily from the one derived in the present work also to the case in which the mobility has the more general form $b(c)=c^{m}(1-c)^{n}$, with $1 \leqslant m<2$ and $n \geqslant 2$. In the case $m \geqslant 2$, the only difference would be that the set $\{\mathbf{x} \in \Omega \mid c(\mathbf{x}, t)=0\}$ would have zero measure for each time $t$, so that no compactly supported solutions of Problem $\mathrm{P}$ would be admitted in this case (see in particular the estimate (49) derived in Section 2.2 and the corollary of Lemma 2 in Elliott and Garcke ${ }^{7}$ ). 
Therefore, the $\mathrm{CH}$ equation we want to analyze degenerates on the set $\{c=0 ; c=1\}$, but the singularity is concentrated on the set $\{c=1\}$ only. This feature introduces a nontrivial difference with respect to the $\mathrm{CH}$ equation analyzed in the literature. For instance, in the degenerate cases studied in Elliott and Garcke ${ }^{7}$ and Schimperna and Zelik, ${ }^{9}$ the degeneracy and the singularity sets coincide and the singularity behavior compensates the degeneracy of the mobility. In Barrett et al, ${ }^{23}$ a finite element approximation of Problem $P$ is studied in the case of a degenerate mobility and a smooth double well potential and in the case of a degenerate mobility and a logarithmic double well potential. In the former case, the positivity condition of the discrete solution is imposed as a constraint through a discrete variational inequality, while, in the latter case, a classical formulation without constraints is studied for the discrete approximation, because the degeneracy and the singularity sets coincide and the positivity property is ensured also at a discrete level. In this case, an acute partitioning of the domain is required to study the existence of a discrete solution (see Theorem 4.1 in Barrett et $\mathrm{al}^{23}$ ).

In our case, we cannot exploit the relationship between $b$ and $\psi$ at 0 to ensure that $c \geqslant 0$ at a discrete level. Therefore, we impose this condition as a constraint and formulate a discrete variational inequality, as done in Barrett et $\mathrm{al}^{23}$ in the case of a degenerate mobility combined with a smooth double well potential. Differently from the cited paper, we have a variational inequality with a singular term in the potential, which we approach via a regularization method. Using properties of convex calculus, we generalize the requirements introduced in the cited paper to guarantee existence of a discrete solution in the case of the singular potential; in particular, in Theorem 4, we do not require an acute partitioning of the domain.

The paper is organized as follows. In Section 2, we study the existence of a weak solution of (4) to (9) and its positivity properties for the cases of spatial dimension $d=1$ and $d=2,3$ separately. In particular, to handle the singular cellular potential, we introduce and study a regularized problem as an intermediate step. In Sections 3 and 4, we introduce a continuous Galerkin finite element approximation of (4) to (9), we show its well posedness and we prove its convergence in one spatial dimension. Section 4 is devoted to the convergence analysis in one space dimension. In Section 5, we present the iterative scheme we have used to solve the discrete variational inequality and we present some numerical experiments in dimensions one and two. We also discuss the growth of the coarsening domains in 2 dimensions. Section 6 contains a discussion of the main results.

\section{1 | Notation and functional setting}

For a given domain $\omega \subset \Omega, d=1,2,3$, we indicate with $L^{p}(\omega), W^{m, p}(\omega), H^{m}(\omega)=W^{m, 2}(\omega)$ and $L^{p}((0, T) ; V)$ the usual Lebesgue, Sobolev, and Bochner spaces, (see, eg, Adams ${ }^{37}$ ), for $p \in[1, \infty]$ and $m \in \mathbb{N}$, endowed with the corresponding canonical norms and seminorms $\|\cdot\|_{m, p, \omega},\|\cdot\|_{m, \omega},|\cdot|_{m, p, \omega}$ and $|\cdot|_{m, \omega}$, respectively. Throughout, $(\cdot, \cdot)_{\omega}$ denotes the standard $L^{2}$ inner product over $\omega$, and $\langle\cdot, \cdot\rangle_{\omega}$ denotes the duality pairing between $\left(H^{1}(\omega)\right)^{\prime}$ and $\left(H^{1}(\omega)\right)$. We omit the index $\omega$ when $\omega=\Omega$. We indicate with $C(\bar{\Omega}), C^{n}\left(I_{1}, I_{2}\right), n \geqslant 0$, and $C_{x, t}^{s_{1}, s_{2}}\left(\bar{\Omega}_{T}\right), 0<s_{1}, s_{2}<1$ the space of continuous functions from $\bar{\Omega}$ to $\mathbb{R}$, the space of $C^{n}$ continuous functions from interval $I_{1} \subset \mathbb{R}$ to interval $I_{2} \subset \mathbb{R}$, and the space of Hölder continuous functions from $\bar{\Omega}_{T}$ to $\mathbb{R}$ with Hölder exponents $s_{1}$ and $s_{2}$ in the arguments $x$ and $t$, respectively. Moreover, we introduce the following set notation: $\{0<q<1\}:=\left\{(\mathbf{x}, t) \in \Omega_{T}: 0<q(\mathbf{x}, t)<1\right\}$, for a given function $q: \Omega_{T} \rightarrow \mathbb{R}$.

Furthermore, $C$ denotes throughout a generic positive constant independent of the unknown variables, the discretization and the regularization parameters, the value of which might change from line to line; $C_{1}, C_{2}, \ldots$ indicate generic positive constants whose particular value must be tracked through the calculations; $C(a)$ denotes a constant depending on the nonnegative parameter $a$, such that, for $C_{1}>0$, if $a \leqslant C_{1}$, there exists a $C_{2}>0$ such that $C(a) \leqslant C_{2}$.

It is useful to introduce the inverse Laplacian operator $\mathcal{G}: \mathcal{F} \rightarrow V$ such that

$$
(\nabla \mathcal{G} v, \nabla \eta)=<v, \eta>\quad \forall \eta \in H^{1}(\Omega)
$$

where $\mathcal{F}=\left\{v \in\left(H^{1}(\Omega)\right)^{\prime}:\langle v, 1>=0\}\right.$ and $V=\left\{v \in H^{1}(\Omega):(v, 1)=0\right\}$. The existence and uniqueness of an element $\mathcal{G} v \in V$, for any $v \in \mathcal{F}$, follows from the Lax-Milgram Theorem and the Poincaré's inequality.

We can define a norm on $\mathcal{F}$ by setting

$$
\left.\|v\|_{\mathcal{F}}:=|\mathcal{G} v|_{1} \equiv<v, \mathcal{G} v\right\rangle^{1 / 2} \quad \forall v \in \mathcal{F} .
$$


We will use the following Sobolev interpolation result, (see, eg, Adams ${ }^{37}$ ). Let $p \in[1, \infty], m \geqslant 1$, we set

$$
r \in \begin{cases}{[p, \infty]} & \text { if } m-\frac{d}{p}>0, \\ {[p, \infty)} & \text { if } m-\frac{d}{p}=0, \\ {\left[p,-\frac{d}{m-(d / p)}\right]} & \text { if } m-\frac{d}{p}<0,\end{cases}
$$

and $\mu=\frac{d}{m}\left(\frac{1}{p}-\frac{1}{r}\right)$. Then there is a constant $C$ such that

$$
\|v\|_{0, r} \leqslant C\|v\|_{0, p}^{1-\mu}\|v\|_{m, p}^{\mu} \quad \forall v \in W^{m, p}(\Omega) .
$$

Let $\mathcal{T}_{h}$ be a quasi-uniform conforming decomposition of $\Omega$ into $d$-simplices $K, d=1,2,3$, and let us introduce the following finite element spaces:

$$
\begin{aligned}
S^{h} & :=\left\{\chi \in C(\bar{\Omega}):\left.\chi\right|_{K} \in P^{1}(K) \forall K \in \mathcal{T}_{h}\right\} \subset H^{1}(\Omega), \\
K^{h} & :=\left\{\chi \in S^{h}: \chi \geqslant 0 \text { in } \Omega\right\},
\end{aligned}
$$

where $\mathbb{P}_{1}(K)$ indicates the space of polynomials of total order one on $K$.

Let $J$ be the set of nodes of $\mathcal{T}_{h}$ and $\left\{x_{j}\right\}_{j \in J}$ be the set of their coordinates. Moreover, let $\left\{\phi_{j}\right\}_{j \in J}$ be the Lagrangian basis functions associated with each node $j \in J$. Denoting by $\pi^{h}: C(\bar{\Omega}) \rightarrow S^{h}$ the standard Lagrangian interpolation operator we define the lumped scalar product as

$$
\left(\eta_{1}, \eta_{2}\right)^{h}=\int_{\Omega} \pi^{h}\left(\eta_{1}(x) \eta_{2}(x)\right) d x \equiv \sum_{j \in J}\left(1, \chi_{j}\right) \eta_{1}\left(x_{j}\right) \eta_{2}\left(x_{j}\right)
$$

for all $\eta_{1}, \eta_{2} \in C(\bar{\Omega})$. We also introduce the $L^{2}$ projection operator $P^{h}: L^{2}(\Omega) \rightarrow S^{h}$ and its lumped version $\hat{P}^{h}: L^{2}(\Omega) \rightarrow S^{h}$ defined by

$$
\begin{aligned}
\left(P^{h} \eta, \chi\right) & =(\eta, \chi) \quad \forall \chi \in S^{h}, \\
\left(\hat{P}^{h} \eta, \chi\right)^{h} & =(\eta, \chi) \quad \forall \chi \in S^{h} .
\end{aligned}
$$

We recall the following well-known results, (see, eg, Barrett et $\mathrm{al}^{23}$ ).

Lemma 1. The following properties hold

$$
\begin{gathered}
|\chi|_{m, p_{2}} \leqslant C h^{-d\left(\frac{1}{p_{1}}-\frac{1}{p_{2}}\right)}|\chi|_{m, p_{1}} \quad \forall \chi \in S^{h}, 1 \leqslant p_{1} \leqslant p_{2} \leqslant \infty, m=0,1 ; \\
|\chi|_{1, p} \leqslant C h^{-1}\|\chi\|_{0, p} \quad \forall \chi \in S^{h}, 1 \leqslant p \leqslant \infty ; \\
\lim _{h \rightarrow 0}\left\|\left(I-\pi^{h}\right) \eta\right\|_{0, \infty}=0 \quad \forall \eta \in C(\bar{\Omega}) ; \\
\left\|\left(I-P^{h}\right) \eta\right\|_{0}+h\left|\left(I-P^{h}\right) \eta\right|_{1} \leqslant C h^{m}|\eta|_{m} \quad \forall \eta \in H^{m}(\Omega), m=1,2 ; \\
\|\chi\|_{0}^{2} \leqslant(\chi, \chi)^{h} \leqslant(d+2)\|\chi\|_{0}^{2} \quad \forall \chi \in S^{h} \\
\left|\left(v^{h}, \chi\right)^{h}-\left(v^{h}, \chi\right)\right| \leqslant C h^{1+m}\left\|v^{h}||_{m}\right\| \chi \|_{1} \quad \forall v^{h}, \chi \in S^{h}, m=0,1 ; \\
\left|\left(I-\pi^{h}\right) \eta\right|_{m, r} \leqslant C h^{1-m}|\eta|_{1, r} \quad \forall \eta \in W^{1, r}(\Omega), m=0,1, r \in[1, \infty] \text { if } d=1 ; \\
\lim _{h \rightarrow 0}\left\|\left(I-\pi^{h}\right) \eta\right\|_{1}=0 \quad \forall \eta \in H^{1}(\Omega) \text { if } d=1 .
\end{gathered}
$$


Similarly to (11), we define the operators $\mathcal{G}^{h}: \mathcal{F} \rightarrow V^{h}$ and $\hat{\mathcal{G}}^{h}: \mathcal{F}^{h} \rightarrow V^{h}$ as follows:

$$
\begin{gathered}
\left(\nabla \mathcal{G}^{h} v, \nabla \chi\right)=<v, \chi>\quad \forall \chi \in S^{h}, \\
\left(\nabla \hat{\mathcal{C}}^{h} v, \nabla \chi\right)=(v, \chi)^{h} \quad \forall \chi \in S^{h},
\end{gathered}
$$

where $\mathcal{F}^{h}=\{v \in \bar{C}(\Omega):(v, 1)=0\}$ and $V^{h}=\left\{v^{h} \in S^{h}:\left(v^{h}, 1\right)=0\right\}$.

\section{2 | EXISTENCE OF WEAK SOLUTIONS}

In this section, we will give the existence proof for different classes of weak solutions in the cases of spatial dimensions $d=1$ and $d=2,3$. To do that, we introduce a suitable regularization of the original problem.

\section{1 | Regularized problem}

Given $\delta \in(0,1)$ and $\epsilon \in(0,1)$, we define a regularized mobility as follows:

$$
b_{\delta, \epsilon}(r):= \begin{cases}b(\delta) & \text { for } r \leqslant \delta, \\ b(r) & \text { for } \delta<r<1-\epsilon, \\ b(1-\epsilon) & \text { for } r \geqslant 1-\epsilon,\end{cases}
$$

for all $r \in \mathbb{R}$. We also define a regularized potential by setting

$$
\psi_{1, \epsilon}^{\prime \prime}(r):= \begin{cases}\psi_{1}^{\prime \prime}(1-\epsilon) & \text { for } r \geqslant 1-\epsilon, \\ \psi_{1}^{\prime \prime}(r) & \text { for } r<1-\epsilon,\end{cases}
$$

for all $r \in \mathbb{R}$. We note that the two parameters $\delta$ and $\epsilon$ could be identified in a single regularization parameter in the analysis. Since in Section 3.1, we will introduce a discrete problem in which only the regularization of the singular potential near $c=1$ will be considered in the form (27), without considering any regularization near $c=0$, we prefer for clarity to separate the regularization parameters for the mobility and the potential around $c=0$ and $c=1$. By expanding $\psi_{1}(r)$ in (8) in a neighborhood of $(1-\epsilon)$ when $r \geqslant 1-\epsilon$, we obtain $\psi_{1, \epsilon}$, ie,

$$
\psi_{1, \epsilon}(r):= \begin{cases}-\left(1-c^{*}\right) \log \epsilon+\frac{3}{2}\left(1-c^{*}\right)-\frac{2}{\epsilon}\left(1-c^{*}\right)(1-r)+\frac{1-c^{*}}{2 \epsilon^{2}}(1-r)^{2} & \text { for } r \geqslant 1-\epsilon, \\ \psi_{1}(r) & \text { for } r<1-\epsilon,\end{cases}
$$

and $\psi_{1, \epsilon}^{\prime}$, ie,

$$
\psi_{1, \epsilon}^{\prime}(r):= \begin{cases}\frac{2}{\epsilon}\left(1-c^{*}\right)-\frac{1-c^{*}}{\epsilon^{2}}(1-r) & \text { for } r \geqslant 1-\epsilon \\ \psi_{1}^{\prime}(r) & \text { for } r<1-\epsilon\end{cases}
$$

Furthermore, expanding $\psi_{1, \epsilon}(\cdot)$ in the Taylor series around $(1-\epsilon)$, with an argument $s>1$ and with $\epsilon<1$, using (27), (28), and (29), we obtain

$$
\begin{aligned}
\psi_{1, \epsilon}(s) & =\psi_{1, \epsilon}(1-\epsilon)+\psi_{1, \epsilon}^{\prime}(1-\epsilon)(s-(1-\epsilon))+\frac{1}{2} \psi_{1, \epsilon}^{\prime \prime}(1-\epsilon)(s-(1-\epsilon))^{2} \\
& =-\left(1-c^{*}\right) \log \epsilon+\frac{1-c^{*}}{\epsilon}(s-(1-\epsilon))+\frac{1-c^{*}}{2 \epsilon^{2}}(s-(1-\epsilon))^{2} \\
& \geqslant \frac{1-c^{*}}{2 \epsilon^{2}}(s-1)^{2} .
\end{aligned}
$$

Hence, we have that

$$
\psi_{1, \epsilon}(s) \geqslant \frac{1-c^{*}}{2 \epsilon^{2}}\left([s-1]_{+}\right)^{2} \quad \forall s \in \mathbb{R}
$$

where $[\cdot]_{+}=\max \{\cdot, 0\}$. 
Introducing the extension $\bar{\psi}_{2} \in C^{2}(\mathbb{R})$ of $\psi_{2} \in C^{2}([0,1])$,

$$
\bar{\psi}_{2}(r):= \begin{cases}\psi_{2}(1)+(r-1) \psi_{2}^{\prime}(1)+\frac{1}{2}(r-1)^{2} \psi_{2}^{\prime \prime}(1) & \text { for } r \geqslant 1, \\ \psi_{2}(r) & \text { for } r \leqslant 1,\end{cases}
$$

we set $\psi_{\epsilon}(r):=\psi_{1, \epsilon}(r)+\bar{\psi}_{2}(r)$. We note that $\psi_{1}(r)+\psi_{2}(r) \geqslant 0$ for $r \leqslant 0$, and that

$$
\psi_{1, \epsilon}(r)+\bar{\psi}_{2}(r)=-\left(1-c^{*}\right) \log \epsilon-\frac{1}{3}+\left[\frac{2}{\epsilon}\left(1-c^{*}\right)+\left(2 c^{*}-3\right)\right](r-1)+\left[\frac{1-c^{*}}{2 \epsilon^{2}}+\frac{c^{*}-3}{2}\right](r-1)^{2}
$$

for $r \geqslant 1$. Since there exists a sufficiently small positive value $\epsilon_{0}$ such that the expressions in the square brackets in (32) are both positive $\forall \epsilon \leqslant \epsilon_{0}$, we obtain that

$$
\psi_{\epsilon}(s) \geqslant-C \quad \forall s \in \mathbb{R}, \epsilon \leqslant \epsilon_{0},
$$

where the constant $C$ is independent of $\epsilon$. Moreover, from (8), (29), and (31), we get that

$$
\left|\psi_{\epsilon}^{\prime}(s)\right| \leqslant C+C|s|+C|s|^{2} \quad \forall s \in \mathbb{R} .
$$

We now introduce the following regularized version of (4) to (6):

Problem $\mathbf{P}_{\delta \epsilon}$. Find $c_{\delta, \epsilon}(\mathbf{x}, t)$ such that

$$
\begin{gathered}
\frac{\partial c_{\delta, \epsilon}}{\partial t}=\operatorname{div}\left(b_{\delta, \epsilon}\left(c_{\delta, \epsilon}\right) \nabla w_{\delta, \epsilon}\right) \quad \text { in } \Omega_{T}, \\
w_{\delta, \epsilon}=-\Delta c_{\delta, \epsilon}+\psi_{\epsilon}^{\prime}\left(c_{\delta, \epsilon}\right) \quad \text { in } \Omega_{T}, \\
c_{\delta, \epsilon}(\mathbf{x}, 0)=c_{0}(\mathbf{x}) \quad \forall \mathbf{x} \in \Omega, \\
\nabla c_{\delta, \epsilon} \cdot v=b_{\delta, \epsilon}\left(c_{\delta, \epsilon}\right) \nabla w_{\delta, \epsilon} \cdot v=0 \quad \text { on } \partial \Omega \times(0, T) .
\end{gathered}
$$

We have the following existence result for $P_{\delta, \epsilon}$.

Theorem 1. Let $\partial \Omega \in C^{1,1}$ or $\Omega$ is convex, with $d=1,2,3$, and suppose that $c_{0} \in H^{1}(\Omega)$. Then for every $0<T<\infty$, there exists a pair $\left(c_{\delta, \epsilon}, w_{\delta, \epsilon}\right)$ such that

$$
\begin{gathered}
c_{\delta, \epsilon} \in L^{\infty}\left(0, T ; H^{1}(\Omega)\right) \cap H^{1}\left(0, T ;\left(H^{1}(\Omega)\right)^{\prime}\right), \\
w_{\delta, \epsilon} \in L^{2}\left(0, T ; H^{1}(\Omega)\right),
\end{gathered}
$$

which satisfies (35) to (38) in the following mixed weak formulation:

$$
\left\{\begin{array}{l}
\int_{0}^{T}\left\langle\frac{\partial c_{\delta, \epsilon}}{\partial t}(t), \xi(t)\right\rangle_{\left(H^{1}\right)^{\prime}, H^{1}}=-\int_{\Omega_{T}} b_{\delta, \epsilon}\left(c_{\delta, \epsilon}\right) \nabla w_{\delta, \epsilon} \nabla \xi, \\
\int_{\Omega} w_{\delta, \epsilon} \phi=\gamma \int_{\Omega} \nabla c_{\delta, \epsilon} \nabla \phi+\int_{\Omega} \psi_{\epsilon}^{\prime}\left(c_{\delta, \epsilon}\right) \phi, \quad \text { a.e. } t \in[0, T],
\end{array}\right.
$$

for all $\xi \in L^{2}\left(0, T ; H^{1}(\Omega)\right), \phi \in H^{1}(\Omega)$, with $c_{\delta, \epsilon}(\boldsymbol{x}, 0)=c_{0}(\boldsymbol{x})$.

Proof. The proof of the theorem comes from Theorem 2 in Elliott and Garcke. ${ }^{7}$ It suffices to note that, from (26) to (33), it follows that $b_{\delta, \epsilon} \in C\left(\mathbb{R}, \mathbb{R}^{+}\right)$, with $b_{1} \leqslant b_{\delta, \epsilon}(r) \leqslant B_{1}, b_{1}, B_{1}>0$, and (33) and (34) imply that $\psi_{\epsilon} \in C^{2}(\mathbb{R}, \mathbb{R}),\left|\psi_{\epsilon}^{\prime}(r)\right| \leqslant$ $C|r|^{2}+C|r|+C, \psi_{\epsilon}(r) \geqslant-C$.

We also get a further regularity, namely,

Lemma 2. In the hypotheses of Theorem 1, we have that $c_{\delta, \epsilon} \in L^{2}\left(0, T ; H^{3}(\Omega)\right)$. The weak solution of (41) thus satisfies the primal weak formulation

$$
\int_{0}^{T}\left\langle\frac{\partial c_{\delta, \epsilon}}{\partial t}, \xi\right\rangle_{\left(H^{1}\right)^{\prime}, H^{1}}=-\int_{\Omega_{T}} b_{\delta, \epsilon}\left(c_{\delta, \epsilon}\right) \nabla\left(-\gamma \Delta c_{\delta, \epsilon}+\psi_{\epsilon}^{\prime}\left(c_{\delta, \epsilon}\right)\right) \nabla \xi,
$$


for all $\xi \in L^{2}\left(0, T ; H^{1}(\Omega)\right)$, with $c_{\delta, \epsilon}(\boldsymbol{x}, 0)=c_{0}(\boldsymbol{x})$.

Proof. From (34), (39), and Sobolev inequality (13), with $m=1, r \in[1,6]$ for $d=3$ and $m=1, r \geqslant 1$ for $d=1,2$, we get that $\psi_{\epsilon}^{\prime} \in L^{\infty}\left(0, T ; L^{2}(\Omega)\right)$. Using then (40) and applying the elliptic regularity theory to the second equation of (41), we get that $c_{\delta, \epsilon} \in L^{2}\left(0, T ; H^{2}(\Omega)\right)$.

We now show that $\nabla \psi_{\epsilon}^{\prime}\left(c_{\delta, \epsilon}\right)=\psi_{\epsilon}^{\prime \prime}\left(c_{\delta, \epsilon}\right) \nabla c_{\delta, \epsilon} \in L^{2}\left(\Omega_{T}\right)$. It is enough to show it in the case $d=3$. Indeed, from (27)-(31), (39) we have that

$$
\int_{0}^{T} \int_{\Omega}\left|\psi_{\epsilon}^{\prime \prime}\left(c_{\delta, \epsilon}\right) \nabla c_{\delta, \epsilon}\right|^{2} \leqslant\left. C|| \nabla c_{\delta, \epsilon}\right|_{L^{2}\left(\Omega_{T}\right)}+C \int_{0}^{T} \int_{\Omega}\left|c_{\delta, \epsilon} \nabla c_{\delta, \epsilon}\right|^{2} \leqslant C+C \int_{0}^{T} \int_{\Omega} c_{\delta, \epsilon}^{2}\left|\nabla c_{\delta, \epsilon}\right|^{2} .
$$

Since $c_{\delta, \epsilon}^{2}\left|\nabla c_{\delta, \epsilon}\right|^{2}$ is a positive measurable function on $\Omega_{T}$, we can apply Tonelli Theorem, and, from the application of (13), with $r=\infty, m=2$, we get

$$
\begin{aligned}
\int_{0}^{T} \int_{\Omega}\left|\psi_{\epsilon}^{\prime \prime}\left(c_{\delta, \epsilon}\right) \nabla c_{\delta, \epsilon}\right|^{2} & \leqslant C+C \int_{0}^{T}\left(\left\|c_{\delta, \epsilon}(t)\right\|_{L^{\infty}(\Omega)}^{2} \int_{\Omega}\left|\nabla c_{\delta, \epsilon}(t, \mathbf{x})\right|^{2}\right) \leqslant C+C \int_{0}^{T}\left(\left.\left\|c_{\delta, \epsilon}(t)\right\|\right|^{1 / 2}\left\|c_{\delta, \epsilon}(t)\right\|_{2,2}^{3 / 2}\left\|\nabla c_{\delta, \epsilon}(t)\right\|^{2}\right) \\
& \leqslant C+C\left\|c_{\delta, \epsilon}\right\|_{L^{\infty}\left(0, T ; H^{1}(\Omega)\right)}^{2} \int_{0}^{T}\left(\left\|c_{\delta, \epsilon}(t)\right\|^{1 / 2}\left\|c_{\delta, \epsilon}(t)\right\|_{2,2}^{3 / 2}\right) .
\end{aligned}
$$

Using (39), the Young's inequality and the fact that $c_{\delta, \epsilon} \in L^{2}\left(0, T ; H^{2}(\Omega)\right)$, we obtain

$$
\int_{0}^{T} \int_{\Omega}\left|\psi_{\epsilon}^{\prime \prime}\left(c_{\delta, \epsilon}\right) \nabla c_{\delta, \epsilon}\right|^{2} \leqslant C+\|\left. c_{\delta, \epsilon}\right|_{L^{2}\left(0, T ; H^{2}(\Omega)\right)} ^{2} \leqslant C,
$$

so that $\nabla \psi_{\epsilon}^{\prime}\left(c_{\delta, \epsilon}\right) \in L^{2}\left(\Omega_{T}\right)$. Finally, since $\nabla w_{\delta, \epsilon} \in L^{2}\left(\Omega_{T}\right)$, we get that $\nabla \Delta c_{\delta, \epsilon} \in L^{2}\left(\Omega_{T}\right)$, and this gives the thesis.

To pass to the limit for $\delta, \epsilon \rightarrow 0$ in the regularized problem (41), we need to obtain a priori estimates uniform in the regularization parameters. This is the aim of the next section.

\section{2 | A priori estimates}

Thanks to Theorem 1 and Lemma 2, we have that $w_{\delta, \epsilon}=-\gamma \Delta c_{\delta, \epsilon}+\psi_{\epsilon}^{\prime}\left(c_{\delta, \epsilon}\right) \in L^{2}\left(0, T ; H^{1}(\Omega)\right)$. Then we can take $\xi \equiv w_{\delta, \epsilon}$ in (42) and obtain, arguing as in Lemma 2 in Elliott and Garcke, ${ }^{7}$ the following result.

Lemma 3. If $0 \leqslant c_{0}<1$ and $c_{0} \in H^{1}(\Omega)$, there exists an $\epsilon_{0}$ such that for all $0<\epsilon \leqslant \epsilon_{0}$ and $\delta>0$ the following estimate holds, for almost all $t \in[0, T]$,

$$
\int_{\Omega}\left(\frac{\gamma}{2}\left|\nabla c_{\delta, \epsilon}(t)\right|^{2}+\psi_{\epsilon}\left(c_{\delta, \epsilon}(t)\right)\right)+\int_{\Omega_{T}} b_{\delta, \epsilon}\left(c_{\delta, \epsilon}\right)\left|\nabla w_{\delta, \epsilon}\right|^{2}=\int_{\Omega}\left(\frac{\gamma}{2}\left|\nabla c_{0}\right|^{2}+\psi_{\epsilon}\left(c_{0}\right)\right) \leqslant C .
$$

Note that, given $0 \leqslant c_{0}<1$, for $\epsilon$ sufficiently small, we have that $\psi_{\epsilon}\left(c_{0}\right) \leqslant \psi\left(c_{0}\right) \in L^{1}(\Omega)$, since $c_{0} \in H^{1}(\Omega) \subset \subset L^{6}(\Omega)$ (in the case $d=3$ ).

We define now the following Entropy function $\Phi_{\delta, \epsilon}$ and its derivative $\Psi_{\delta, \epsilon}$,

$$
\Psi_{\delta, \epsilon}(r):=\int_{A}^{r} \frac{d s}{b_{\delta, \epsilon}(s)} ; \quad \Phi_{\delta, \epsilon}(r):=\int_{A}^{r} \Psi_{\delta, \epsilon}(s) d s ; \quad \Phi_{\delta, \epsilon}^{\prime \prime}(r)=\Psi_{\delta, \epsilon}^{\prime}(r)=\frac{1}{b_{\delta, \epsilon}(r)},
$$

with $A>1$. We note that

$$
\Psi_{\delta, \epsilon}(r) \leqslant 0, \quad \Phi_{\delta, \epsilon}(r) \geqslant 0, \quad \text { for } r \leqslant A,
$$

and that

$$
\Phi_{\delta, \epsilon}(r) \leqslant \Phi(r), \quad \text { for } 0 \leqslant r \leqslant 1,
$$

where $\Phi(r)=\lim _{\delta \rightarrow 0, \epsilon \rightarrow 0} \Phi_{\delta, \epsilon}(r)$. We define also $\Psi(r)=\lim _{\delta \rightarrow 0, \epsilon \rightarrow 0} \Psi_{\delta, \epsilon}(r)$, and observe that, for $0<r \leqslant A$, 


$$
\begin{gathered}
\Psi(r)=\frac{1}{1-r}-\log (1-r)+\log (r)-C, \\
\Phi(r)=\log \left(\frac{1}{1-r}\right)-r[\log (1-r)-1]+\log (1-r)+r \log (r)-r+C .
\end{gathered}
$$

Since $\Phi_{\delta, \epsilon}^{\prime \prime}$ is bounded, we can take $\xi \equiv \Phi_{\delta, \epsilon}^{\prime}\left(c_{\delta, \epsilon}\right) \in L^{2}\left(0, T ; H^{1}(\Omega)\right)$ in (42) and obtain, following again Lemma 2 in Elliott and Garcke, ${ }^{7}$ the following result.

Lemma 4. If $0 \leqslant c_{0}<1$ almost everywhere in $\Omega$, for almost all $t \in[0, T]$ and for $\delta, \epsilon>0$ the following estimate holds:

$$
\int_{\Omega} \Phi_{\delta, \epsilon}\left(c_{\delta, \epsilon}(t)\right)+\gamma \int_{\Omega_{T}}\left|\Delta c_{\delta, \epsilon}\right|^{2}+\int_{\Omega_{T}} \psi_{1, \epsilon}^{\prime \prime}\left|\nabla c_{\delta, \epsilon}\right|^{2}=\int_{\Omega} \Phi_{\delta, \epsilon}\left(c_{0}\right)-\int_{\Omega_{T}} \bar{\psi}_{2}^{\prime \prime}\left|\nabla c_{\delta, \epsilon}\right|^{2} \leqslant C .
$$

Note from (46) and (48) that $\int_{\Omega} \Phi_{\delta, \epsilon}\left(c_{0}\right) \leqslant \int_{\Omega} \Phi\left(c_{0}\right) \leqslant C$ if $0 \leqslant c_{0}<1$ almost everywhere in $\Omega$.

\subsection{Passage to the limit for the case $d=1$}

Using the a priori estimates (43) and (49), we can take the limit for $\delta, \epsilon \rightarrow 0$ in (41) and obtain the following theorem.

Theorem 2. Let $d=1$ and $c_{0} \in H^{1}(\Omega)$, with $0 \leqslant c_{0}<1$. Then there exist a subsequence of $\left(c_{\delta, \epsilon}, w_{\delta, \epsilon}\right)$ and functions $c \in L^{\infty}\left(0, T ; H^{1}(\Omega)\right) \cap H^{1}\left(0, T ;\left(H^{1}(\Omega)\right)^{\prime}\right) \cap C_{x, t}^{\frac{1}{2}, \frac{1}{8}}\left(\bar{\Omega}_{T}\right)$ and $w \in L_{l o c}^{2}(0<c<1)$ with $\frac{\partial w}{\partial x} \in L_{l o c}^{2}(0<c<1)$, such that, for $\delta, \epsilon \rightarrow 0$,

$$
\begin{gathered}
c_{\delta, \epsilon} \rightarrow c \text { weakly in } L^{2}\left(0, T ; H^{1}(\Omega)\right), \\
c_{\delta, \epsilon} \rightarrow c \text { uniformly on } \bar{\Omega}_{T}, \\
w_{\delta, \epsilon} \rightarrow w, \quad \frac{\partial w_{\delta, \epsilon}}{\partial x} \rightarrow \frac{\partial w}{\partial x} \quad \text { weakly in } L_{l o c}^{2}(0<c<1) .
\end{gathered}
$$

Moreover, we have

$$
0 \leqslant c<1 \text { a.e. in } \Omega_{T} .
$$

The limit point $(c, w)$ satisfies the weak formulation

$$
\left\{\begin{array}{l}
\int_{0}^{T}\left\langle\frac{\partial c}{\partial t}, \eta\right\rangle d t+\int_{0<c<1}\left(b(c) \frac{\partial w}{\partial x}, \frac{\partial \eta}{\partial x}\right) d t=0 \\
\int_{0<c<1} \gamma\left(\frac{\partial c}{\partial x}, \frac{\partial \theta}{\partial x}\right) d t+\int_{0<c<1}\left(\psi^{\prime}(c), \theta\right) d t-\int_{0<c<1}(w, \theta) d t=0,
\end{array}\right.
$$

for all $\eta, \theta \in L^{2}\left(0, T ; H^{1}(\Omega)\right)$, with $c(\cdot, 0)=c_{0}(\cdot)$.

Proof. The proof follows Bernis and Friedman, ${ }^{38}$ with suitable modifications since we are working with a dual mixed formulation of Problem P instead of a primal formulation.

From (43) and from Poincaré's inequality, we get that $c_{\delta, \epsilon} \in L^{2}\left(0, T ; H^{1}(\Omega)\right)$, from which (50) follows. Since $d=1$, from (43), Poincaré's inequality and Sobolev embedding results we get that $c_{\delta, \epsilon}$ is uniformly bounded on $\bar{\Omega}_{T}$, and that its $C_{x, t}^{\frac{1}{2}, \frac{1}{8}}\left(\bar{\Omega}_{T}\right)$ norm is uniformly bounded (see Bernis and Friedman ${ }^{38}$ for details). Hence, every sequence $c_{\delta, \epsilon}$ is uniformly bounded and equicontinuous on $\bar{\Omega}_{T}$, and, by the Ascoli-Arzelá Theorem, we get (51).

To prove that $c \geqslant 0$ in $\Omega_{T}$, let us suppose that there exists a point $(\bar{x}, \bar{t}) \in \Omega_{T}$ such that $c(\bar{x}, \bar{t})<0$. From the uniform continuity of $c_{\delta, \epsilon}$ and $c$ and from the uniform convergence (51), we get that there exist $\gamma_{1}, \gamma_{2}$ and $\left(\delta_{0}, \epsilon_{0}\right)$ such that

$$
c_{\delta, \epsilon}(x, \bar{t})<-\gamma_{1} \quad \text { if }|x-\bar{x}|<\gamma_{2}, \delta \leqslant \delta_{0}, \epsilon \leqslant \epsilon_{0} .
$$

Hence, from (45) and (46), we get

$$
\Phi_{\delta, \epsilon}\left(c_{\delta, \epsilon}(x, \bar{t})\right)=\int_{A}^{c_{\delta, \epsilon}(x, \bar{t})} \Psi_{\delta, \epsilon}(s) d s \geqslant-\int_{-\gamma_{1}}^{0} \Psi_{\delta, \epsilon}(s) d s .
$$


Since $0 \leqslant-\Psi_{\delta^{\prime}, \epsilon^{\prime}}(r) \leqslant-\Psi_{\delta^{\prime \prime}, \epsilon^{\prime \prime}}(r)$ for $-\gamma_{1} \leqslant r \leqslant 0$ when $\delta^{\prime \prime} \leqslant \delta^{\prime}, \epsilon^{\prime \prime} \leqslant \epsilon^{\prime}$, by applying the Beppo Levi Theorem and due to the presence of the term $\log (r)$ in $(47)$, we get

$$
\lim _{\delta \rightarrow 0, \epsilon \rightarrow 0} \Phi_{\delta, \epsilon}\left(c_{\delta, \epsilon}(x, \bar{t})\right) \geqslant-\int_{-\gamma_{1}}^{0} \Psi(s) d s=\infty .
$$

Using the fact that $\Phi_{\delta, \epsilon}(r) \geqslant 0$ for $r \leqslant A$, we get that

$$
\lim _{\delta \rightarrow 0, \epsilon \rightarrow 0} \int_{\Omega} \Phi_{\delta, \epsilon}\left(c_{\delta, \epsilon}(x, \bar{t})\right) d x=\infty,
$$

which contradicts (49). Hence $c \geqslant 0$ in $\Omega_{T}$. With a similar argument and due to the presence of the term $\log (1-r)$ in (47), we can show that $c \leqslant 1$ in $\Omega_{T}$. Thanks to the presence of the term $\log (1-r)$ in (48) or, equivalently, in (28), we prove that, for each $t \in[0, T]$, the set $\{x \in \Omega \mid c(x, t)=1\}$ has measure zero. Indeed, suppose that there exists a $\bar{t} \in[0, T]$ such that the set $S:=\{x \in \Omega \mid c(x, \bar{t})=1\}$ has positive measure. From the uniform continuity of $c_{\delta, \epsilon}$ and $c$ and from the uniform convergence (51), we get that there exist a modulus of continuity $\gamma_{1}(\epsilon)$, with $\gamma_{1}(0)=0$, and $\left(\delta_{0}, \epsilon_{0}\right)$ such that

$$
c_{\delta, \epsilon}(x, \bar{t}) \geqslant 1-\gamma_{1}(\epsilon) \quad \forall x \in S, \delta \leqslant \delta_{0}, \epsilon \leqslant \epsilon_{0} .
$$

From (28), we have that

$$
\psi_{1, \epsilon}\left(c_{\delta, \epsilon}(x, \bar{t})\right) \geqslant-\left(1-c^{*}\right) \log \gamma_{1}(\epsilon)-\frac{2}{\gamma_{1}(\epsilon)}\left(1-c^{*}\right), \forall x \in S .
$$

Using the fact that $\psi_{1, \epsilon}(r) \geqslant 0$ for $r \geqslant 0$ if $\epsilon$ is sufficiently small, we get that

$$
\limsup _{\delta \rightarrow 0, \epsilon \rightarrow 0} \int_{\Omega} \psi_{1, \epsilon}\left(c_{\delta, \epsilon}(x, \bar{t})\right) d x \geqslant \limsup _{\epsilon \rightarrow 0}\left(-\left(1-c^{*}\right) \log \gamma_{1}(\epsilon)-\frac{2}{\gamma_{1}(\epsilon)}\left(1-c^{*}\right)\right)|S| \rightarrow \infty,
$$

which contradicts (43). Hence, $0 \leqslant c<1$ almost everywhere in $\Omega_{T}$.

We now show the boundedness of $w_{\delta, \epsilon}$ and $\frac{\partial w_{\delta, \epsilon}}{\partial x}$ on $L_{\mathrm{loc}}^{2}(\{0<c<1\})$. For any $\eta>0$, we set

$$
\begin{aligned}
D_{\eta}^{+} & =\left\{(x, t) \in \bar{\Omega}_{T}: \eta<c(x, t)<1\right\}, \\
D_{\eta}^{+}(t) & =\{x \in \bar{\Omega}: \eta<c(x, t)<1\} .
\end{aligned}
$$

On account of the uniform convergence (51), for a fixed $\eta>0$, it follows that there exist $\bar{\delta}, \bar{\epsilon}$ such that, for all $\delta \leqslant \bar{\delta}, \epsilon \leqslant \bar{\epsilon}$,

$$
\begin{aligned}
& \left|c_{\delta, \epsilon}\right|<2 \eta \quad \forall(x, t) \notin D_{\eta}^{+}, \\
& \frac{1}{8} \eta \leqslant c_{\delta, \epsilon} \quad \forall(x, t) \in D_{\frac{\eta}{4}}^{+} .
\end{aligned}
$$

From (43) and from (55) we have

$$
b_{\delta, \epsilon, \min }\left(\frac{\eta}{8}\right) \int_{D_{\frac{\eta}{4}}^{+}}\left|\frac{\partial w_{\delta, \epsilon}}{\partial x}\right|^{2} d x d t \leqslant \int_{D_{\frac{\eta}{4}}^{+}} b\left(c_{\delta, \epsilon}\right)\left|\frac{\partial w_{\delta, \epsilon}}{\partial x}\right|^{2} d x d t \leqslant C,
$$

where $b_{\delta, \epsilon, \min }(\eta):=\min _{\eta \leqslant z<1} b_{\delta, \epsilon}(z)$.

We now introduce a cutoff function $\theta_{\eta} \in C_{0}^{\infty}\left(D_{\frac{\eta}{4}}^{+}\right)$such that

$$
\theta_{\eta}(\cdot, t) \equiv 1 \quad \text { on } D_{\frac{\eta}{2}}^{+}(t), \quad 0 \leqslant \theta_{\eta}(\cdot, t) \leqslant 1 .
$$

Noting that, since $c \in C_{x, t}^{\frac{1}{2}, \frac{1}{8}}\left(\bar{\Omega}_{T}\right)$, we have that $C \eta \leqslant\left|x_{2}-x_{1}\right|^{1 / 2}$ for $x_{1}, x_{2} \in D_{\frac{\eta}{4}}^{+} \backslash D_{\frac{\eta}{2}}^{+}$, then we can choose a $\theta_{\eta}(\cdot, t)$ such that

$$
\left|\nabla \theta_{\eta}(\cdot, t)\right| \leqslant C \eta^{-2} \text {. }
$$

Since $\theta_{\eta}^{2} w_{\delta, \epsilon} \in H^{1}(\Omega)$, we can take $\phi=\theta_{\delta}^{2} w_{\delta, \epsilon}$ in (41) and integrate over time in the interval $(0, T)$. On account of the uniform convergence (51), given $c(x, t)<1$, we have $c_{\delta, \epsilon}(x, t)<1$ for sufficiently small $\delta$ and $\epsilon$; hence, $\frac{1}{8} \eta \leqslant c_{\delta, \epsilon}<1$ on $D_{\frac{n}{4}}^{+}$, and thus $\psi_{\epsilon}^{\prime}\left(c_{\delta, \epsilon}\right)$ is bounded. We obtain

$$
\begin{aligned}
\int_{\Omega_{T}} \theta_{\eta}^{2}\left(w_{\delta, \epsilon}\right)^{2} d x d t & \leqslant C\left\|c_{\delta, \epsilon}\right\|_{L^{2}\left(0, T ; H^{1}(\Omega)\right)}\left\|\frac{\partial}{\partial x}\left(\theta_{\eta}^{2} w_{\delta, \epsilon}\right)\right\|_{L^{2}\left(\Omega_{T}\right)}+C\left\|\psi_{\epsilon}^{\prime}\left(c_{\delta, \epsilon}\right)\right\|_{L^{2}\left(\Omega_{T}\right)}\left\|\theta_{\eta} w_{\delta, \epsilon}\right\|_{L^{2}\left(\Omega_{T}\right)} \\
& \leqslant C\left(1+\eta^{-2}\right)\left\|\theta_{\eta} w_{\delta, \epsilon}\right\|_{L^{2}\left(\Omega_{T}\right)}+C\left\|\frac{\partial w_{\delta, \epsilon}}{\partial x}\right\|_{L^{2}\left(D_{\frac{\eta}{4}}^{+}\right)} .
\end{aligned}
$$


Using now Young's inequality and (56), we infer

$$
\int_{\Omega_{T}} \theta_{\eta}^{2}\left(w_{\delta, \epsilon}\right)^{2} d x d t \leqslant C+C\left(\eta^{-4}\right)
$$

Therefore, combining (56) and (59) and recalling the definition of $\theta_{\eta}(\cdot, t)$, we have that, for all $\eta>0$,

$$
\left\|w_{\delta, \epsilon}\right\|_{L^{2}\left(0, T ; H^{1}\left(D_{\frac{\eta}{4}}^{+}(t)\right)\right)} \leqslant C+C\left(\eta^{-4}\right) .
$$

Applying (60) on compact subsets of the set $\{0<c<1\} \equiv D_{0}^{+}$, we eventually obtain (52).

From the boundedness of $c_{\delta, \epsilon}$ on $\Omega_{T}$ and from (43), we get that $b\left(c_{\delta, \epsilon}\right) \frac{\partial w_{\delta, \epsilon}}{\partial x} \in L^{2}\left(\Omega_{T}\right)$; hence, from (41), we deduce that

$$
\partial_{t} c_{\delta, \epsilon} \rightarrow \partial_{t} c \text { weakly in } L^{2}\left(0, T ;\left(H^{1}(\Omega)\right)^{\prime}\right) \text {. }
$$

Finally, we pass to the limit for $\delta, \epsilon \rightarrow 0$ in each term in (41), to show that we can extract a subsequence of the approximating functions $\left(c_{\delta, \epsilon}, w_{\delta, \epsilon}\right.$ ), which converges to a solution of (54). Using (50), (52), and (61), we can pass to the limit for $\delta, \epsilon \rightarrow 0$ in the first term of the first equation of (41) and in the first and second terms in the second equation of (41), obtaining easily the corresponding terms in (54). From the facts that $\psi_{1, \epsilon}(\cdot), \bar{\psi}_{2}(\cdot) \in C^{2}(\mathbb{R}, \mathbb{R})$, that $\psi_{\epsilon}(r) \rightarrow \psi(r)$ uniformly for $r<1$, from the uniform convergence (51) and the fact that $c<1$ almost everywhere in $\Omega_{T}$, we have that $\psi_{\epsilon}\left(c_{\delta, \epsilon}\right) \rightarrow \psi(c)$ almost everywhere in $\Omega_{T}$. We can thus pass to the limit for $\delta, \epsilon \rightarrow 0$ in the third term in the second equation of (41), obtaining the corresponding limit term in (54). Finally, we can rewrite the second term in the first equation of (41) as

$$
\int_{\Omega_{T}} b_{\delta, \epsilon}\left(c_{\delta, \epsilon}\right) \nabla w_{\delta, \epsilon} \nabla \xi=\int_{\Omega_{T} \backslash D_{\frac{n}{4}}^{+}} b_{\delta, \epsilon}\left(c_{\delta, \epsilon}\right) \nabla w_{\delta, \epsilon} \nabla \xi+\int_{D_{\frac{\eta}{4}}^{+}}\left[b_{\delta, \epsilon}\left(c_{\delta, \epsilon}\right)-b(c)\right] \nabla w_{\delta, \epsilon} \nabla \xi+\int_{D_{\frac{n}{4}}^{+}} b(c) \nabla w_{\delta, \epsilon} \nabla \xi .
$$

Taking the limit for $\delta, \epsilon \rightarrow 0$ in the right-hand side of (62), we use (43), the Cauchy-Schwarz inequality and the first inequality in (55) to bound the first term; we moreover use the uniform convergence (51), together with the fact that $b_{\delta, \epsilon}(\cdot), b(\cdot) \in C^{0}(\mathbb{R}, \mathbb{R}),(56)$, and the Cauchy-Schwarz inequality to bound the second term. Using (52), we obtain, for $\eta \rightarrow 0$, the corresponding limit term in the weak formulation (54)

Finally, from the uniform convergence $(51)$, we have that $c(\cdot, 0)=c_{0}(\cdot)$.

\subsection{Passage to the limit for the case $d=2,3$}

In the present case, we do not have the uniform convergence of the regularized solution as a consequence of Sobolev embedding theorems. Hence, the term $\nabla w_{\delta, \epsilon}$ might not have a limit in $L^{2}\left(\Omega_{T}\right)$; therefore, we use the entropy estimate (49) to identify a suitable class of weak solutions, for which we can take the limit for $\delta, \epsilon \rightarrow 0$ in (41). More precisely, we have the following theorem.

Theorem 3. Let $d=2,3$ and $c_{0} \in H^{1}(\Omega)$, with $0 \leqslant c_{0}<1$ almost everywhere in $\Omega$. Then there exists a subsequence of $\left(c_{\delta, \epsilon}, J_{\delta, \epsilon}\right)$, with $J_{\delta, \epsilon}:=b_{\delta, \epsilon}\left(c_{\delta, \epsilon}\right) \nabla w_{\delta, \epsilon}$, and functions $c \in L^{\infty}\left(0, T ; H^{1}(\Omega)\right) \cap H^{1}\left(0, T ;\left(H^{1}(\Omega)\right)^{\prime}\right) \cap L^{2}\left(0, T ; H^{2}(\Omega)\right)$ and $J \in L^{2}\left(\Omega_{T} ; \mathbb{R}^{n}\right)$, such that, for $\delta, \epsilon \rightarrow 0$,

$$
\begin{gathered}
c_{\delta, \epsilon} \rightarrow \text { c strongly in } L^{2}\left(0, T ; H^{1}(\Omega)\right), \\
\partial_{t} c_{\delta, \epsilon} \rightarrow \partial_{t} c \text { weakly in } L^{2}\left(0, T ;\left(H^{1}(\Omega)\right)^{\prime}\right), \\
\Delta c_{\delta, \epsilon} \rightarrow \Delta c \quad \text { weakly in } L^{2}\left(\Omega_{T}\right), \\
J_{\delta, \epsilon} \rightarrow J \quad \text { weakly in } L^{2}\left(\Omega_{T}\right) .
\end{gathered}
$$

Moreover, we have

$$
0 \leqslant c<1 \text { a.e. in } \Omega_{T} \text {. }
$$


The limit point (c,J) satisfies the weak formulation

$$
\left\{\begin{array}{l}
\int_{0}^{T}\left\langle\frac{\partial c}{\partial t}, \xi\right\rangle d t=\int_{\Omega_{T}} J \cdot \nabla \xi, \quad \forall \xi \in L^{2}\left(0, T ; H^{1}(\Omega)\right), \\
\int_{\Omega_{T}} J \cdot \eta=-\int_{\Omega_{T}}\left[\gamma \Delta c \nabla \cdot(b(c) \eta)+b \psi^{\prime \prime}(c) \nabla c \cdot \eta\right]
\end{array} \quad \forall \eta \in L^{2}\left(0, T ; H^{1}\left(\Omega ; \mathbb{R}^{n}\right)\right),\right.
$$

with $\eta \cdot v=0$ on $\partial \Omega \times(0, T), c(\cdot, 0)=c_{0}(\cdot)$, and with $\nabla c \cdot v=0$ on $\partial \Omega$.

Proof. The proof is an adaptation of the proof of Theorem 1 in Elliott and Garcke. ${ }^{7}$ From (49) and from elliptic regularity, we have (65) and the fact that $c_{\delta, \epsilon} \in L^{2}\left(0, T ; H^{2}(\Omega)\right.$ ). From (43), we get (66), which also implies (64) from (41). Moreover, recalling compactness results, we have that $L^{\infty}\left(0, T ; H^{1}(\Omega)\right) \cap H^{1}\left(0, T ;\left(H^{1}(\Omega)\right)^{\prime}\right) \cap L^{2}\left(0, T ; H^{2}(\Omega)\right)$ is compactly embedded in $C^{0}\left(0, T ; L^{6}(\Omega) \cap L^{2}\left(0, T ; H^{1}(\Omega)\right.\right.$ ) (for $d=3$; see, eg, Adams $\left.{ }^{37}\right)$, we obtain $(63)$ and $c(\cdot, 0)=c_{0}(\cdot)$.

To prove that $c \geqslant 0$ almost everywhere in $\Omega_{T}$, let us suppose that there exists a set $S \subset \Omega_{T}$ with positive measure such that $c<0$ on $S$. Note that, since $c \in C^{0}\left([0, T] ; L^{1}(\Omega)\right)$, this hypothesis can be formally obtained starting from a set $E$ with positive measure for which $f_{E} c d x<0$ and using the Lebesgue Theorem (see Theorem 1.2 in Grün ${ }^{22}$ for details). On the other hand, from (63), we have that $c_{\delta, \epsilon}$ converges strongly to $c$ in $L^{2}\left(\Omega_{T}\right)$, we can apply the Egorov Theorem and obtain that, for each $\gamma>0$, there exists a subset $S^{\prime}$, with $\left|S \backslash S^{\prime}\right|<\gamma$, on which (a subsequence of) $c_{\delta, \epsilon}$ converges uniformly to $c$. Hence, we get that there exist $\gamma_{1}$ and $\left(\delta_{0}, \epsilon_{0}\right)$ such that

$$
c_{\delta, \epsilon}(x, t)<-\gamma_{1} \quad \text { on } S^{\prime}, \delta \leqslant \delta_{0}, \epsilon \leqslant \epsilon_{0} .
$$

Therefore, using the fact that $\Phi_{\delta, \epsilon}(r) \geqslant 0$ for $r \leqslant A$, we get that

$$
\int_{\Omega_{T}} \Phi_{\delta, \epsilon}\left(c_{\delta, \epsilon}(x, t)\right) d t d x \geqslant \int_{S^{\prime}} \int_{A}^{c_{\delta, \epsilon}(x, t)} \Psi_{\delta, \epsilon}(s) d s \geqslant-\left|S^{\prime}\right| \int_{-\gamma_{1}}^{0} \Psi_{\delta, \epsilon}(s) d s .
$$

Applying the Beppo Levi Theorem and due to the presence of the term $\log (r)$ in (47), we get that

$$
\lim _{\delta \rightarrow 0, \epsilon \rightarrow 0} \int_{\Omega_{T}} \Phi_{\delta, \epsilon}\left(c_{\delta, \epsilon}(x, t)\right) d x=\infty,
$$

which contradicts (49). Hence, $c \geqslant 0$ almost everywhere in $\Omega_{T}$. Using a similar argument and exploiting the presence of the term $\log (1-r)$ in (47), we can show that $c \leqslant 1$ almost everywhere in $\Omega_{T}$. Suppose now that there exists a set $V \subset \Omega_{T}$, with positive measure, on which $c(x, t)=1$. Applying the Egorov theorem, we obtain that, for each $\gamma>0$, there exists a subset $V^{\prime}$, with $\left|V \backslash V^{\prime}\right|<\gamma$, on which (a subsequence of) $c_{\delta, \epsilon}$ converges uniformly to $c$. Hence, we get that there exists a modulus of continuity $\gamma_{1}(\epsilon)$, with $\gamma_{1}(0)=0$, and $\left(\delta_{0}, \epsilon_{0}\right)$ such that

$$
c_{\delta, \epsilon} \geqslant 1-\gamma_{1}(\epsilon) \text { on } V^{\prime}, \delta \leqslant \delta_{0}, \epsilon \leqslant \epsilon_{0} .
$$

From (28), we have that

$$
\psi_{1, \epsilon}\left(c_{\delta, \epsilon}(x, t)\right) \geqslant-\left(1-c^{*}\right) \log \gamma_{1}(\epsilon)-\frac{2}{\gamma_{1}(\epsilon)}\left(1-c^{*}\right), \forall(x, t) \in V^{\prime} .
$$

Using again the Beppo Levi theorem and the fact that $\psi_{1, \epsilon}(r) \geqslant 0$ for $r \geqslant 0$ if $\epsilon$ is sufficiently small, we get that

$$
\limsup _{\delta \rightarrow 0, \epsilon \rightarrow 0} \int_{\Omega} \psi_{1, \epsilon}\left(c_{\delta, \epsilon}(x, \bar{t})\right) d x \geqslant \limsup _{\epsilon \rightarrow 0}\left(-\left(1-c^{*}\right) \log \gamma_{1}(\epsilon)-\frac{2}{\gamma_{1}(\epsilon)}\left(1-c^{*}\right)\right)\left|V^{\prime}\right| \rightarrow \infty,
$$

which contradicts (43). Hence, $0 \leqslant c<1$ almost everywhere in $\Omega_{T}$.

We now prove that the limit point satisfies the weak formulation (68). Starting from (41) and using (64) and (66), we identify in the limit the first equation in (68). Since, from Lemma 2 , we have that $c_{\delta, \epsilon} \in L^{2}\left(0, T ; H^{3}(\Omega)\right.$ ), we can write

$$
\int_{\Omega_{T}} J_{\delta, \epsilon} \cdot \eta=\int_{\Omega_{T}} b_{\delta, \epsilon}\left(c_{\delta, \epsilon}\right) \nabla\left(-\gamma \Delta c_{\delta, \epsilon}+\psi_{\epsilon}^{\prime}\left(c_{\delta, \epsilon}\right)\right) \eta,
$$

with $\left.\eta \in L^{2}\left(0, T ; H^{1}\left(\Omega, \mathbb{R}^{n}\right)\right) \cap L^{\infty}\left(\Omega_{T}, \mathbb{R}^{n}\right)\right)$, and $\eta \cdot \nu=0$ on $\partial \Omega \times(0, T)$. We can then integrate by parts the first term on the right-hand side, obtaining 


$$
\int_{\Omega_{T}} J_{\delta, \epsilon} \cdot \eta=\int_{\Omega_{T}}\left[\gamma \Delta c_{\delta, \epsilon} \nabla \cdot\left(b_{\delta, \epsilon}\left(c_{\delta, \epsilon}\right) \eta\right)+b_{\delta, \epsilon}\left(c_{\delta, \epsilon}\right) \psi_{\epsilon}^{\prime \prime}\left(c_{\delta, \epsilon}\right) \nabla c_{\delta, \epsilon} \cdot \eta\right]
$$

From (66), we have that the first term in (70) converges to the first term in the second equation in (68). From (63) and (65), the facts that $b_{\delta, \epsilon}(\cdot) \rightarrow b(\cdot)$ uniformly and that $b_{\delta, \epsilon}^{\prime}(\cdot)$ is bounded, and from the Lebesgue theorem, we have that the second term in (70) converges to the second term in the second equation in (68). (We do not report the details of calculation here, which are the same as those in Elliott and Garcke, ${ }^{7}$ proof of Theorem 1). Concerning the last term in (70), we write

$$
b_{\delta, \epsilon}\left(c_{\delta, \epsilon}\right) \psi_{\epsilon}^{\prime \prime}\left(c_{\delta, \epsilon}\right)=b_{\delta, \epsilon}\left(c_{\delta, \epsilon}\right) \psi_{1, \epsilon}^{\prime \prime}\left(c_{\delta, \epsilon}\right)+b_{\delta, \epsilon}\left(c_{\delta, \epsilon}\right) \bar{\psi}_{2}^{\prime \prime}\left(c_{\delta, \epsilon}\right)
$$

Consider the second term on the right-hand side of $(71)$. Recalling that $b_{\delta, \epsilon}(\cdot) \rightarrow b(\cdot)$ uniformly and, from compactness results, $c_{\delta, \epsilon} \rightarrow c$ strongly in $C^{0}\left(0, T ; L^{6}(\Omega)\right.$ ) (for $d=3$ ), from (31) and the fact that $c<1$ almost everywhere in $\Omega_{T}$, we have that

$$
b_{\delta, \epsilon}\left(c_{\delta, \epsilon}\right) \bar{\psi}_{2}^{\prime \prime}\left(c_{\delta, \epsilon}\right) \rightarrow b(c) \psi_{2}^{\prime \prime}(c) \quad \text { a.e. in } \Omega_{T}
$$

Since $0 \leqslant c<1$ almost everywhere in $\Omega_{T}$, we can pass to the limit in the corresponding term in the second term on the right-hand side of (70), using (63), (72), and the Vitali convergence theorem. For the first term on the right-hand side of (71), we note that $b_{\delta, \epsilon}\left(c_{\delta, \epsilon}\right) \psi_{1, \epsilon}^{\prime \prime}\left(c_{\delta, \epsilon}\right)$ is uniformly bounded and that

$$
b_{\delta, \epsilon}\left(c_{\delta, \epsilon}\right) \psi_{1, \epsilon}^{\prime \prime}\left(c_{\delta, \epsilon}\right) \rightarrow b(c) \psi_{1}^{\prime \prime}(c) \quad \text { a.e. in } \Omega_{T} .
$$

Hence, we can pass to the limit in the corresponding term in the second term on the right-hand side of (70), using (63) and the Lebesgue theorem. We finally obtain that the last term in (70) converges to the last term in the second equation in (68).

\section{3 | CONTINUOUS GALERKIN FINITE ELEMENT APPROXIMATION SCHEME}

In this section, we introduce the finite element and time discretization of (4) to (6). The entropy estimate (49), which guarantees the positivity of the solution, is not straightforwardly available at the discrete level. Indeed, given $\chi \in S^{h}$, we have that $\nabla\left(P^{h}[\Psi(\chi)]\right) \neq \frac{1}{b(\chi)} \nabla \chi$, where $\Psi$ has been defined in (47). In Grün, ${ }^{24}$ a suitable approximation of the mobility $\bar{b}$ has been introduced such that

$$
\bar{b}(\chi) \nabla\left(P^{h}[\Psi(\chi)]\right)=\nabla \chi,
$$

which consists of an harmonic average of the mobility on a structured mesh.

Following Barrett et al, ${ }^{23}$ we impose this property as a constraint through a variational inequality. In the sequel, we will show that the solution of our discrete formulation, for the discretization parameters tending to zero, satisfies the mixed weak formulation (54) and is thus consistent.

We set $\Delta t=T / N$ for a $N \in \mathbb{N}$ and $t_{n}=n \Delta t, n=, \ldots, N$. For $d=1,2,3$, starting from a datum $c_{0} \in H^{1}(\Omega)$ and $c_{h}^{0}=\pi^{h} c_{0}$ (if $d=1$ ) or $c_{h}^{0}=\hat{P}^{h} c_{0}$ (if $d=2,3$ ), with $0 \leqslant c_{h}^{0}<1$, we consider the following fully discretized problem:

Problem $\mathbf{P}^{h}$. For $n=1, \ldots, N$, given $c_{h}^{n-1} \in K^{h}$, find $\left(c_{h}^{n}, w_{h}^{n}\right) \in K^{h} \times S^{h}$ such that for all $(\chi, \phi) \in S^{h} \times K^{h}$,

$$
\left\{\begin{array}{l}
\left(\frac{c_{h}^{n}-c_{h}^{n-1}}{\Delta t}, \chi\right)^{h}+\left(b\left(c_{h}^{n-1}\right) \nabla w_{h}^{n}, \nabla \chi\right)=0, \\
\gamma\left(\nabla c_{h}^{n}, \nabla\left(\phi-c_{h}^{n}\right)\right)+\left(\psi_{1}^{\prime}\left(c_{h}^{n}\right), \phi-c_{h}^{n}\right)^{h} \geqslant\left(w_{h}^{n}-\psi_{2}^{\prime}\left(c_{h}^{n-1}\right), \phi-c_{h}^{n}\right)^{h} .
\end{array}\right.
$$

Remark 1. Choosing $\phi \equiv 0$ and $\phi \equiv 2 c_{h}^{n}$ in (74) yields, for all $j \in J$, that either $\left|c_{h}^{n}\left(x_{j}\right)\right|=0$ or $\left|c_{h}^{n}\left(x_{j}\right)\right|>0$ and $\gamma\left(\nabla c_{h}^{n}, \nabla \chi_{j}\right)+\left(\psi_{1}^{\prime}\left(c_{h}^{n}\right)+\psi_{2}^{\prime}\left(c_{h}^{n-1}\right)-w_{h}^{n}, \chi_{j}\right)^{h}=0$. 
Defining the discrete energy functional $F_{1}: S^{h} \rightarrow \mathbb{R}^{+}$as

$$
F_{1}\left[c_{h}^{n}\right]=\int_{\Omega}\left\{\frac{\gamma}{2}\left|\nabla c_{h}^{n}\right|^{2}+\psi_{1}\left(c_{h}^{n}\right)+\chi_{\mathbb{R}^{+}}\left(c_{h}^{n}\right)\right\} d x,
$$

where $\chi_{\mathbb{R}^{+}}(\cdot)$ is the indicator function of the closed and convex set $\mathbb{R}^{+}$, and endowing the space $S^{h}$ with the lumped scalar product (14), we can rewrite the second equation of System 74 as

$$
\left(w_{h}^{n}-\psi_{2}^{\prime}\left(c_{h}^{n-1}\right), \phi-c_{h}^{n}\right)^{h}+F_{1}\left[c_{h}^{n}\right] \leqslant F_{1}[\phi], \quad \forall \phi \in S^{h},
$$

which is equivalent to

$$
w_{h}^{n}-\psi_{2}^{\prime}\left(c_{h}^{n-1}\right) \in \partial F_{1}\left[c_{h}^{n}\right]
$$

where $\partial$ is the subdifferential of the convex and lower semicontinuous function $F_{1}$. We note that the formulation (77) represents the generalized discrete analogous of the subdifferential approach to the standard $\mathrm{CH}$ equation with constraints introduced in Kenmochi et al..$^{39}$ Here, that approach is generalized to our case. Inequality 76 will be used in (100) and (110) to study the convergence of a suitable regularized problem to the original one. In particular, using the properties of convex and lower semicontinuous functions and of subdifferential calculus, we avoid the necessity to bound the first derivative of the potential $\psi_{1}(\cdot)$, like in Barrett et $\mathrm{al}^{23}{ }^{23}$ where it requires to introduce the hypothesis of acuteness of the partition of the domain.

Remark 2. Given the assumption $0 \leqslant c_{h}^{0}<1$, the term $\left(\psi_{1}^{\prime}\left(c_{h}^{n}\right), \chi-c_{h}^{n}\right)^{h}$ in the second equation of (74) is well defined, since we will show that $\left|c_{h}^{0}\right|_{0, \infty}<1$ implies that $\left|c_{h}^{n}\right|_{0, \infty}<1$ for all $n \geqslant 1$ (see Lemma 7). From now on, we assume that $0 \leqslant c_{h}^{0}<1$. Notice that this is a physically consistent assumption, since subregions in the domains where the cellular phase concentrates against infinite cell-cell repulsion are unphysical.

We now introduce the discrete Green operator of the first degenerate elliptic equation in (74), which will be used to express the chemical potential $w_{h}^{n}$ in terms of $\frac{c_{h}^{n}-c_{h}^{n-1}}{\Delta t}$ and to show the well posedness of Problem $\mathrm{P}^{h}$. We follow the approach in Barrett et $\mathrm{al}^{23}$ to invert the degenerate elliptic form on a proper closed and convex subset of $S^{h}$.

To introduce the subset of $S^{h}$ on which we can invert the degenerate elliptic form $\left(b\left(c_{h}^{n-1}\right) \nabla w_{h}^{n}, \nabla \chi\right)$, we must subdivide the partition $\mathcal{T}^{h}$ of $\Omega$ into elements on which $c_{h}^{n-1} \equiv 0$ and elements on which $c_{h}^{n-1} \neq 0$. Given $q^{h} \in K^{h}$ with $f q^{h} \in(0,1)$, where $f q^{h}=\frac{1}{|\Omega|}\left(q^{h}, 1\right)$, we define the set of passive nodes $J_{0}\left(q^{h}\right) \subset J$ by

$$
j \in J_{0}\left(q^{h}\right) \Longleftrightarrow \hat{P}^{h} q^{h}\left(x_{j}\right)=0 \Longleftrightarrow\left(q^{h}, \chi_{j}\right)=0 .
$$

The nodes in the set $J_{+}\left(q^{h}\right)=J \backslash J_{0}\left(q^{h}\right)$ are called active nodes; these nodes can be partitioned into mutually disjoint and maximally connected subsets $I_{m}\left(q^{h}\right)$ such that $J_{+}\left(q^{h}\right) \equiv \cup_{m=1}^{M} I_{m}\left(q^{h}\right)$. In Figure 2, we show a possible partition of the domain in regions where $q_{h} \equiv 0$ (the colored region) and $q_{h} \neq 0$. We note that the node $j \in J_{0}\left(q^{h}\right)$, and all other nodes are in $J_{+}\left(q^{h}\right)$.

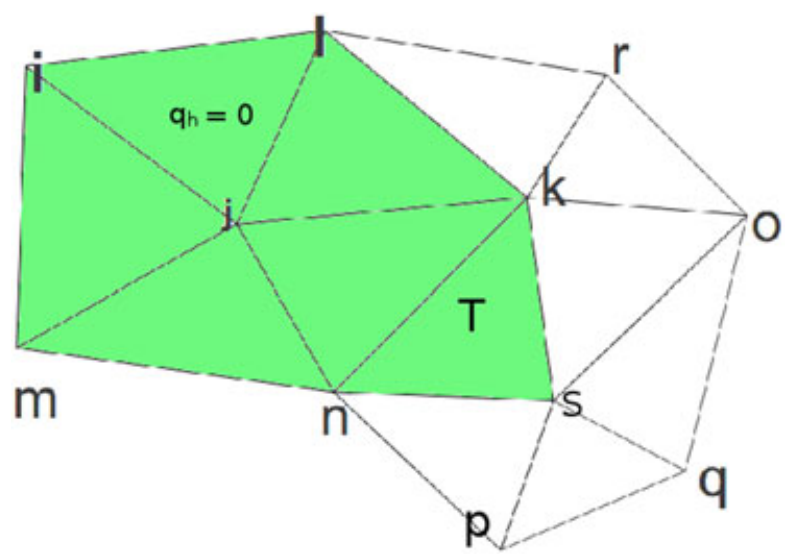

FIGURE 2 A partition of the domain $\Omega$ into regions where $q_{h} \equiv 0$ (the colored region) and $q_{h} \neq 0$ [Colour figure can be viewed at wileyonlinelibrary.com] 
Defining

$$
\Sigma_{m}\left(q^{h}\right)=\sum_{j \in I_{m}\left(q^{h}\right)} \chi_{j}
$$

we note that

$$
\Sigma_{m}\left(q^{h}\right) \equiv 1 \quad \text { on each element on which } q^{h} \neq 0,
$$

since all the vertices of this elements belong to $I_{m}\left(q^{h}\right)$. Note that there are also elements on which $q^{h} \equiv 0$ and $\Sigma_{m}\left(q^{h}\right) \equiv 1$, like the element $T$ in Figure 2. The presence of these elements complicate the treatment of the problem and seems to be neglected in the proofs in Barrett et al, ${ }^{23}$ whereas, here, they will be considered in the calculations of the following estimates. Hence, on each element $K \in \mathcal{T}^{h}$, we have that $q^{h} \equiv 0$ or $\Sigma_{m}\left(q^{h}\right) \equiv 1$ for some $m$, except for those elements on which both $q^{h} \equiv 0$ and $\Sigma_{m}\left(q^{h}\right) \equiv 1$. Let us define the following sets:

$$
\Omega_{m}\left(q^{h}\right)=\left\{\bigcup_{K \in \mathcal{T}^{h}} \bar{K}: \Sigma_{m}\left(q^{h}\right)(x)=1 \forall x \in K\right\},
$$

ie, the union of the maximally connected elements on which $q^{h} \neq 0$, or $q^{h} \equiv 0$ and the indexes of the vertices of the elements belong to $I_{m}\left(q^{h}\right)$ for a given $m$. We also set

$$
\Gamma_{m}\left(q^{h}\right)=\operatorname{supp}\left\{\Sigma_{m}\left(q^{h}\right)\right\} \backslash \Omega_{m}\left(q^{h}\right) .
$$

Finally, we introduce the space

$$
V^{h}\left(q^{h}\right)=\left\{v^{h} \in S^{h}: v^{h}\left(x_{j}\right)=0 \forall j \in J_{0}\left(q^{h}\right) \text { and }\left(v^{h}, \Sigma_{m}\left(q^{h}\right)\right)^{h}=0, m=1, \ldots, M\right\},
$$

that consists of all $v^{h} \in S^{h}$, which are orthogonal (with respect to the lumped discrete scalar product (14)) to $\chi_{j}$, for $j \in J_{0}\left(q^{h}\right)$, (see (78)), and that have zero average (again with respect to the scalar product (14)) on each element, which does not contain any passive node.

We recall from Barrett et $\mathrm{al}^{23}$ that any $v^{h} \in S^{h}$ can be written as

$$
v^{h} \equiv \bar{v}^{h}+\sum_{j \in J_{0}\left(q^{h}\right)} v^{h}\left(x_{j}\right) \chi_{j}+\sum_{m=1}^{M}\left[f_{\Omega_{m}\left(q^{h}\right)} v^{h}\right] \Sigma_{m}\left(q^{h}\right),
$$

where $\bar{v}^{h}$ is the $\hat{P}^{h}$ projection of $v^{h}$ onto $V^{h}\left(q^{h}\right)$, and

$$
f_{\Omega_{m}\left(q^{h}\right)} v^{h}:=\frac{\left(v^{h}, \Sigma_{m}\left(q^{h}\right)\right)^{h}}{\left(1, \Sigma_{m}\left(q^{h}\right)\right)} .
$$

We can now define, for all $q^{h} \in K^{h}$ with $q^{h}<1$, the discrete anisotropic Green's operator $\hat{G}_{q^{h}}^{h}: V^{h}\left(q^{h}\right) \rightarrow V^{h}\left(q^{h}\right)$ as

$$
\left(b\left(q^{h}\right) \nabla \hat{G}_{q^{h}}^{h} h^{h}, \nabla \chi\right)=\left(v^{h}, \chi\right)^{h} \quad \forall \chi \in S^{h} .
$$

The well posedness of $\hat{G}_{q^{h}}^{h}$ can be shown as in Barrett et $\mathrm{al}^{23}$ (see in particular formulas 2.23 and 2.24 in Barrett et $\mathrm{al}^{23}$ ), choosing $\chi=\chi_{j}$ for $j \in J_{0}\left(q^{h}\right)$ and $\chi=\Sigma_{m}\left(q^{h}\right)$, for $m=1, \ldots, M$, and using the fact that $\left\|b\left(q^{h}\right)\right\|_{0, \infty} \leqslant C$ for all $q^{h} \in S^{h}$.

We now study a regularized version of Problem 74, to deal with the singularity in the cellular potential and to show the well posedness of Problem 74 when the regularization parameter tends to zero.

\section{1 | Regularized problem}

To show the well posedness of Problem $\mathrm{P}^{h}$, we introduce the following regularized version of (74):

Problem $\mathbf{P}_{\varepsilon}^{h}$. For $n=1, \ldots, N$, given $c_{h}^{n-1} \in K^{h}$, with $c_{h}^{n-1}<1$ and $\left|c_{h}^{n-1}\right|_{1} \leqslant C$, find $\left(c_{h, \epsilon}^{n}, w_{h, \epsilon}^{n}\right) \in K^{h} \times S^{h}$ such that for all $(\chi, \phi) \in S^{h} \times K^{h}$,

$$
\left\{\begin{array}{l}
\left(\frac{c_{h, \epsilon}^{n}-c_{h}^{n-1}}{\Delta t}, \chi\right)^{h}+\left(b\left(c_{h}^{n-1}\right) \nabla w_{h, \epsilon}^{n}, \nabla \chi\right)=0, \\
\gamma\left(\nabla c_{h, \epsilon}^{n}, \nabla\left(\phi-c_{h, \epsilon}^{n}\right)\right)+\left(\psi_{1, \epsilon}^{\prime}\left(c_{h, \epsilon}^{n}\right), \phi-c_{h, \epsilon}^{n}\right)^{h} \geqslant\left(w_{h, \epsilon}^{n}-\bar{\psi}_{2}^{\prime}\left(c_{h}^{n-1}\right), \phi-c_{h, \epsilon}^{n}\right)^{h} .
\end{array}\right.
$$


The following result shows that Problem $\mathrm{P}_{\varepsilon}^{h}$ is well posed.

Lemma 5. There exists a solution $\left(c_{h, \epsilon}^{n}, w_{h, \epsilon}^{n}\right)$ to Problem $P_{\varepsilon}^{h}$. Moreover, the solution $\left\{c_{h, \epsilon}^{n}\right\}_{n=1}^{N}$ is unique, and $w_{h, \epsilon}^{n}$ is unique on $\Omega_{m}\left(c_{h}^{n-1}\right)$, for $m=1, \ldots, M$ and $n=1, \ldots, N$.

Proof. From the first equation in (84) and from (83), it follows that, given $c_{h}^{n-1} \in K^{h}, c_{h}^{n-1}<1$, we search for $c_{h, \epsilon}^{n} \in$ $K^{h}\left(c_{h}^{n-1}\right)$, where

$$
K^{h}\left(c_{h}^{n-1}\right)=\left\{\chi \in K^{h}: \chi-c_{h}^{n-1} \in V^{h}\left(c_{h}^{n-1}\right)\right\} .
$$

Moreover, a solution $w_{h, \epsilon}^{n} \in S^{h}$ can be expressed in terms of $c_{h, \epsilon}^{n}-c_{h}^{n-1}$ through the discrete anisotropic Green operator (83), recalling (81), as

$$
w_{h, \epsilon}^{n}=-\hat{\mathcal{C}}_{c_{h}^{n-1}}^{h}\left[\frac{c_{h, \epsilon}^{n}-c_{h}^{n-1}}{\Delta t}\right]+\sum_{j \in J_{0}\left(c_{h}^{n-1}\right)} \mu_{j, \epsilon}^{n} \chi_{j}+\sum_{m=1}^{M} \lambda_{m, \epsilon}^{n} \Sigma_{m}\left(c_{h}^{n-1}\right),
$$

where $\left\{\mu_{j, \epsilon}^{n}\right\}_{j \in J_{0}\left(c_{h}^{n-1}\right)}$ and $\left\{\lambda_{m, \epsilon}^{n}\right\}_{m=1}^{M}$ are constants which express the values of $w_{h, \epsilon}^{n}$ on the passive nodes and its average value on $\Omega_{m}\left(c_{h}^{n-1}\right)$, respectively. Hence, Problem $\mathrm{P}_{\varepsilon}^{h}$ can be restated as follows: given $c_{h}^{n-1} \in K^{h}$, with $c_{h}^{n-1}<1$, find $c_{h, \epsilon}^{n} \in K^{h}\left(c_{h}^{n-1}\right)$ and constant Lagrange multipliers $\left\{\mu_{j, \epsilon}^{n}\right\}_{j \in J_{0}\left(c_{h}^{n-1}\right)}$ and $\left\{\lambda_{m, \epsilon}^{n}\right\}_{m=1}^{M}$ such that, for all $\chi \in K^{h}$,

$$
\begin{aligned}
& \gamma\left(\nabla c_{h, \epsilon}^{n}, \nabla\left(\chi-c_{h, \epsilon}^{n}\right)\right)+\left(\hat{\mathcal{G}}_{c_{h}^{n-1}}^{h}\left[\frac{c_{h, \epsilon}^{n}-c_{h}^{n-1}}{\Delta t}\right]+\psi_{1, \epsilon}^{\prime}\left(c_{h, \epsilon}^{n}\right), \chi-c_{h, \epsilon}^{n}\right)^{h} \\
\geqslant & \left(\sum_{j \in J_{0}\left(c_{h}^{n-1}\right)} \mu_{j, \epsilon}^{n} \chi_{j}+\sum_{m=1}^{M} \lambda_{m, \epsilon}^{n} \Sigma_{m}\left(c_{h}^{n-1}\right)-\bar{\psi}_{2}^{\prime}\left(c_{h}^{n-1}\right), \chi-c_{h, \epsilon}^{n}\right)^{h} .
\end{aligned}
$$

We note that (87) represents, together with $c_{h, \epsilon}^{n} \in K^{h}\left(c_{h}^{n-1}\right)$, the Karush-Kuhn-Tucker optimality conditions (see, eg, chapter 5 in Ekeland and Temam $^{40}$ ) of the minimization problem

$$
\begin{aligned}
\inf _{v_{h, \epsilon} \in S^{h}} \sup _{\mu_{j, \epsilon}, \lambda_{m, \epsilon}, v_{\epsilon} \geqslant 0}\{ & \gamma\left|v_{h, \epsilon}\right|_{1}^{2}+\frac{1}{\Delta t}\left\|\left[b\left(c_{h}^{n-1}\right)\right]^{1 / 2} \nabla \hat{\mathcal{G}}_{c_{h}^{n-1}}^{h}\left(v_{h, \epsilon}-c_{h}^{n-1}\right)\right\|_{0}^{2} \\
& +2\left(\psi_{1, \epsilon}\left(v_{h, \epsilon}\right)+\bar{\psi}_{2}^{\prime}\left(c_{h}^{n-1}\right) v_{h, \epsilon}, 1\right)^{h}-\sum_{j \in J_{0}\left(c_{h}^{n-1}\right)} \mu_{j, \epsilon}\left(\chi_{j}, v_{h, \epsilon}\right)^{h}-\sum_{m=1}^{M} \lambda_{m, \epsilon}\left(\Sigma_{m}\left(c_{h}^{n-1}\right), v_{h, \epsilon}\right)^{h} \\
& \left.-\left(v_{\epsilon}, v_{h, \epsilon}\right)^{h}\right\},
\end{aligned}
$$

being $v_{\epsilon} \in K^{h}$ the Lagrange multiplier of the inequality constraint. Noting the convexity of $\psi_{1, \epsilon}(\cdot)$ and the fact that $c_{h}^{n-1} \in K^{h}$, the primal form associated to the Lagrangian (88) is a convex, proper, lower semicontinuous, and coercive function from the closed and convex set $K^{h}\left(c_{h}^{n-1}\right)$ to $\mathbb{R}$, and the primal problem is stable. Hence, from the Kuhn-Tucker Theorem (see, eg, theorem 5.1 in Ekeland and $\operatorname{Temam}^{40}$ ), we infer the existence of $c_{h, \epsilon}^{n} \in K^{h}\left(c_{h}^{n-1}\right)$, solution to the primal problem, and Lagrange multipliers $\left\{\mu_{j, \epsilon}^{n}\right\}_{j \in J_{0}\left(c_{h}^{n-1}\right)},\left\{\lambda_{m, \epsilon}^{n}\right\}_{m=1}^{M}$ and $v_{\epsilon}\left(x_{i}\right) \in-\partial \chi_{\mathbb{R}^{+}}\left(c_{h, \epsilon}^{n}\left(x_{i}\right)\right)$, for each $i \in J$ and each $n$. Therefore, from (86) we have the existence of a solution $\left(c_{h, \epsilon}^{n}, w_{h, \epsilon}^{n}\right)_{n=1}^{N}$ to Problem $\mathrm{P}_{\varepsilon}^{h}$.

Let us now prove uniqueness. If, for fixed $n \geqslant 1$, (87) has 2 solutions

$\left(c_{h, \epsilon}^{n, i},\left\{\mu_{j, \epsilon}^{n, i}\right\}_{j \in J_{0}\left(c_{h}^{n-1}\right)},\left\{\lambda_{m, \epsilon}^{n, i}\right\}_{m=1}^{M}\right), i=1,2$, by choosing $\chi=c_{h, \epsilon}^{n, 2}$ in the inequality for $c_{h, \epsilon}^{n, 1}$ and $\chi=c_{h, \epsilon}^{n, 1}$ in the inequality for $c_{h, \epsilon}^{n, 2}$, taking the difference between the 2 inequalities, setting $c_{h, \epsilon}^{n, 1}-c_{h, \epsilon}^{n, 2}=d_{h, \epsilon}^{n} \in V^{h}\left(c_{h}^{n-1}\right)$ and recalling (80), we have

$$
\gamma\left|d_{h, \epsilon}^{n}\right|_{1}^{2}+\frac{1}{\Delta t}\left\|\left[b\left(c_{h}^{n-1}\right)\right]^{1 / 2} \nabla \hat{\mathcal{C}}_{c_{h}^{n-1}}^{h} d_{h, \epsilon}^{n}\right\|_{0}^{2}+\left(\psi_{1, \epsilon}^{\prime}\left(c_{h, \epsilon}^{n, 1}\right)-\psi_{1, \epsilon}^{\prime}\left(c_{h, \epsilon}^{n, 2}\right), d_{h, \epsilon}^{n}\right)^{h} \leqslant 0
$$

so that

$$
\gamma\left|d_{h, \epsilon}^{n}\right|_{1}^{2}+\frac{1}{\Delta t}\left\|\left[b\left(c_{h}^{n-1}\right)\right]^{1 / 2} \nabla \hat{C}_{c_{h}^{n-1}}^{h} d_{h, \epsilon}^{n}\right\|_{0}^{2} \leqslant 0
$$


where we have used the monotonicity of $\psi_{1, \epsilon}^{\prime}(\cdot)$ in the second step. Therefore, the uniqueness of $c_{h, \epsilon}^{n}$ follows from the Poincaré's inequality and the fact that $f c_{h, \epsilon}^{n}=f c_{h, \epsilon}^{0}$. Choosing $\chi=c_{h, \epsilon}^{n} \pm \delta \pi_{h}\left[c_{h, \epsilon}^{n} \Sigma_{m}\left(c_{h}^{n-1}\right)\right]$ in $(87)$, for any $\delta \in(0,1)$ and $m=1, \ldots, M$, yields uniqueness of the Lagrange multiplier $\lambda_{m, \epsilon}^{n}$. Hence, the uniqueness of $w_{h, \epsilon}^{n}$ follows from (86). The proof is complete.

To pass to the limit as $\epsilon \rightarrow 0$ in system (84), we need to deduce suitable $\epsilon$-independent bounds for the solution $\left(c_{h, \epsilon}^{n}, w_{h, \epsilon}^{n}\right)$. The following result holds.

Lemma 6. For every sequence $\epsilon \rightarrow 0$, there exist a subsequence $\epsilon^{\prime} \rightarrow 0$ and $a c_{h}^{n} \in K^{h}$ such that

$$
c_{h, \epsilon^{\prime}}^{n} \rightarrow c_{h}^{n} \quad \text { and } \quad \nabla c_{h, \epsilon^{\prime}}^{n} \rightarrow \nabla c_{h}^{n} \quad \text { for } \epsilon^{\prime} \rightarrow 0
$$

For every sequence $\epsilon \rightarrow 0$, there exist a subsequence $\epsilon^{\prime} \rightarrow 0$ and $a w_{h}^{n} \in S^{h}$ such that

$$
w_{h, \epsilon^{\prime}}^{n} \rightarrow w_{h}^{n} \quad \text { on } \quad \Omega_{m, *}\left(c_{h}^{n-1}\right) \quad \text { and } \quad \nabla w_{h, \epsilon^{\prime}}^{n} \rightarrow \nabla w_{h}^{n} \quad \text { on } \Omega_{m, *}\left(c_{h}^{n-1}\right) \quad \text { for } \epsilon^{\prime} \rightarrow 0,
$$

where $\Omega_{m, *}\left(c_{h}^{n-1}\right)$ is the set of those elements of $\Omega_{m}\left(c_{h}^{n-1}\right)$ on which $c_{h}^{n-1} \not \equiv 0$.

Proof. We start by proving stability bounds for the regularized problem (84). Choosing $\chi=w_{h, \epsilon}^{n}$ in the first equation of (84) and $\phi=c_{h}^{n-1}$ in the second equation of (84), we get

$$
\gamma\left(\nabla c_{h, \epsilon}^{n}, \nabla\left(c_{h, \epsilon}^{n}-c_{h}^{n-1}\right)\right)+\left(\psi_{1, \epsilon}^{\prime}\left(c_{h, \epsilon}^{n}\right)+\bar{\psi}_{2}^{\prime}\left(c_{h}^{n-1}\right), c_{h, \epsilon}^{n}-c_{h}^{n-1}\right)^{h}+\Delta t\left\|\left[b\left(c_{h}^{n-1}\right)\right]^{1 / 2} \nabla w_{h, \epsilon}^{n}\right\|_{0}^{2} \leqslant 0 .
$$

Using now the identity $2 s(s-r)=s^{2}-r^{2}+(s-r)^{2}, \forall r, s \in \mathbb{R}$, and the convexity and the concavity properties of $\psi_{1, \epsilon}(\cdot)$ and $\bar{\psi}_{2}(\cdot)$, it follows that

$$
\frac{\gamma}{2}\left|c_{h, \epsilon}^{n}\right|_{1}^{2}+\frac{\gamma}{2}\left|c_{h, \epsilon}^{n}-c_{h}^{n-1}\right|_{1}^{2}+\left(\psi_{\epsilon}\left(c_{h, \epsilon}^{n}\right), 1\right)^{h}+\Delta t||\left[b\left(c_{h}^{n-1}\right)\right]^{1 / 2} \nabla w_{h, \epsilon}^{n}||_{0}^{2} \leqslant\left(\psi_{\epsilon}\left(c_{h}^{n-1}\right), 1\right)^{h}+\frac{\gamma}{2}\left|c_{h}^{n-1}\right|_{1}^{2} \leqslant C .
$$

From (91) and (33), we deduce that

$$
\left(\left[c_{h, \epsilon}^{n}-1\right]_{+}^{2}, 1\right)^{h} \leqslant C \epsilon^{2}
$$

Hence, from (92), (16), and (20), it follows that

$$
\left\|\left[c_{h, \epsilon}^{n}-1\right]_{+}\right\|_{0, \infty} \leqslant C h^{-d / 2} \epsilon .
$$

Let us suppose that there exist nodes $x_{j}, j \in J$, such that $c_{h, \epsilon}^{n}\left(x_{j}\right) \geqslant 1-\epsilon$. From (28), (91), and (92), we have that

$$
\left(\left[-\left(1-c^{*}\right) \log \epsilon\right] \sum_{j \in J} \chi_{j}, 1\right)+\left.\left(\psi_{1}\left(c_{h, \epsilon}^{n}\right), 1\right)^{h}\right|_{c_{h, \epsilon}^{n}<1-\epsilon} \leqslant C .
$$

Calling $\Omega_{\epsilon}$ the support of the base functions corresponding to nodes on which $c_{h, \epsilon}^{n} \geqslant 1-\epsilon$, using (16) and the fact that $\psi_{1}\left(c_{h, \epsilon}^{n}\right) \geqslant 0$ for all $c_{h, \epsilon}^{n} \geqslant 0$, we have that

$$
\left\|-\left(1-c^{*}\right) \log \epsilon\right\|_{0, \infty, \Omega_{\epsilon}} \leqslant C h^{-3 d / 2},
$$

where the bound is independent on $\epsilon$, which is absurd. Therefore, we deduce that there exists a value $\epsilon_{0}$ sufficiently small such that, for each $\epsilon \leqslant \epsilon_{0}$,

$$
c_{h, \epsilon}^{n}<1
$$


From (91), the fact that $\left(c_{h, \epsilon}^{n}, 1\right)^{h}=\left(c_{h}^{n-1}, 1\right)^{h}$ and (21), from the Poincaré's inequality and the Bolzano-Weierstrass theorem, it follows that there exists a subsequence $\left\{c_{h, \epsilon^{\prime}}^{n}\right\}$ and a $c_{h}^{n} \in K^{h}$ such that (89) holds.

We next show (90). Using the Poincaré's inequality on $\Omega_{m, *}\left(c_{h}^{n-1}\right),(20)$ and (91) leads to

$$
\begin{aligned}
\left(\left(\left[\left(I-f_{\Omega_{m, *}\left(c_{h}^{n-1}\right)}\right) w_{h, \epsilon}^{n}\right] \Sigma_{m}^{*}\left(c_{h}^{n-1}\right)\right)^{2}, 1\right)^{h} & \leqslant C \int_{\Omega_{m, *}\left(c_{h}^{n-1}\right)} \nabla\left|w_{h, \epsilon}^{n}\right|^{2} d x \leqslant C\left[b_{\min }\left(c_{h}^{n-1}\right)\right]^{-1} \int_{\Omega_{m, *}\left(c_{h}^{n-1}\right)} b\left(c_{h}^{n-1}\right) \nabla\left|w_{h, \epsilon}^{n}\right|^{2} d x \\
& \leqslant C\left((\Delta t)^{-1}\right)\left[b_{\min }\left(c_{h}^{n-1}\right)\right]^{-1},
\end{aligned}
$$

where $\Sigma_{m}^{*}\left(c_{h}^{n-1}\right):=\sum_{j \in I_{m}^{*}}\left(c_{h}^{n-1}\right) \chi_{j}$, with $I_{m}^{*}\left(c_{h}^{n-1}\right)$ the subset of nodes of $I_{m}\left(c_{h}^{n-1}\right)$ which are in $\Omega_{m, *}\left(c_{h}^{n-1}\right)$, and

$$
b_{\min }\left(c_{h}^{n-1}\right):=\min _{K \subset \Omega_{m, *}\left(c_{h}^{n-1}\right)} \frac{1}{|K|} \int_{K} b\left(c_{h}^{n-1}\right) d x
$$

We now bound $f_{\Omega_{m, *}}\left(c_{h}^{n-1}\right) w_{h, \epsilon}^{n}$. Let us take

$$
K^{h} \ni \phi=c_{h, \epsilon}^{n}+\Sigma_{m}^{*}\left(c_{h}^{n-1}\right)
$$

in the second equation of system (84). We get

$$
\left(w_{h, \epsilon}^{n}, \Sigma_{m}^{*}\left(c_{h}^{n-1}\right)\right)^{h} \leqslant \gamma\left(\nabla c_{h, \epsilon}^{n}, \nabla \Sigma_{m}^{*}\left(c_{h}^{n-1}\right)\right)+\left(\psi_{1, \epsilon}^{\prime}\left(c_{h, \epsilon}^{n}\right), \Sigma_{m}^{*}\left(c_{h}^{n-1}\right)\right)^{h}+\left(\bar{\psi}_{2}^{\prime}\left(c_{h}^{n-1}\right), \Sigma_{m}^{*}\left(c_{h}^{n-1}\right)\right)^{h} .
$$

Observing that $\Sigma_{m}^{*}\left(c_{h}^{n-1}\right) \equiv 1$ on $\Omega_{m, *}\left(c_{h}^{n-1}\right)$ and that $\bar{\psi}_{2}^{\prime}\left(c_{h}^{n-1}\right)$ is bounded, using moreover (91) and (95), we obtain

$$
\begin{aligned}
\left|\left(w_{h, \epsilon}^{n}, \Sigma_{m}^{*}\left(c_{h}^{n-1}\right)\right)^{h}\right| & \leqslant \gamma\left|\int_{\Gamma_{m}^{*}\left(c_{h}^{n-1}\right)} \nabla c_{h, \epsilon}^{n} \cdot \nabla \Sigma_{m}^{*}\left(c_{h}^{n-1}\right) d x\right|+\left|\left(\psi_{1, \epsilon}^{\prime}\left(c_{h, \epsilon}^{n}\right), \Sigma_{m}^{*}\left(c_{h}^{n-1}\right)\right)^{h}\right|+C|| \Sigma_{m}^{*}\left(c_{h}^{n-1}\right) \|_{0, \infty} \\
& \leqslant \gamma\left|\Gamma_{m}^{*}\left(c_{h}^{n-1}\right)\right|^{1 / 2}|| \nabla \Sigma_{m}^{*}\left(c_{h}^{n-1}\right)\left\|_{0, \infty}\left|c_{h, \epsilon}^{n}\right|_{1}+\left|\left(\psi_{1, \epsilon}^{\prime}\left(c_{h, \epsilon}^{n}\right), \Sigma_{m}^{*}\left(c_{h}^{n-1}\right)\right)^{h}\right|+C|| \Sigma_{m}^{*}\left(c_{h}^{n-1}\right)\right\|_{0, \infty} \\
& \leqslant C h^{-1}+C+\left|\left(\psi_{1, \epsilon}^{\prime}\left(c_{h, \epsilon}^{n}\right), \Sigma_{m}^{*}\left(c_{h}^{n-1}\right)\right)^{h}\right| \leqslant C h^{-1}+C,
\end{aligned}
$$

where $\Gamma_{m}^{*}\left(c_{h}^{n-1}\right):=\operatorname{supp}\left\{\Sigma_{m}^{*}\left(c_{h}^{n-1}\right)\right\} \backslash \Omega_{m, *}\left(c_{h}^{n-1}\right)$.

Now, combining (96) with (97), recalling the definition (82) and using the Poincaré's inequality, we obtain

$$
\left(w_{h, \epsilon}^{n} \Sigma_{m}^{*}\left(c_{h}^{n-1}\right), w_{h, \epsilon}^{n} \Sigma_{m}^{*}\left(c_{h}^{n-1}\right)\right)^{h} \leqslant C+C h^{-1}+C\left((\Delta t)^{-1}\right)\left[b_{\min }\left(c_{h}^{n-1}\right)\right]^{-1} .
$$

Finally, from (98), (91), (21), and the Bolzano-Weierstrass theorem, it follows that there exists a subsequence $\left\{w_{h, \epsilon^{\prime}}^{n}\right\}$ and a $w_{h}^{n} \in S^{h}$ such that (90) holds. The proof is complete.

Lemma 7. The limit point $c_{h}^{n}$ of Lemma 6 satisfies the property $0 \leqslant c_{h}^{n}<1$.

Proof. Passing to the limit for $\epsilon^{\prime} \rightarrow 0$ in (91), using the convergence property (89) and (95) and considering the logarithmic term in $\psi_{1}(\cdot)$, we obtain that $0 \leqslant c_{h}^{n}<1$.

\section{2 | Well posedness of Problem $P^{h}$}

We now have all the ingredients to show the well posedness of Problem $\mathrm{P}^{h}$.

Theorem 4. Let $\Omega \subset \mathbb{R}^{d}, d=1,2,3$, and let $c_{h}^{0} \in K^{h}$ with $c_{h}^{0}<1$ and $\left|c_{h}^{0}\right|_{1} \leqslant C$. Then there exists a solution $\left(c_{h}^{n}\right.$, $\left.w_{h}^{n}\right)$ to Problem (74) for any $n=1, \ldots, N$. Moreover, the solution $\left\{c_{h}^{n}\right\}_{n=1}^{N}$ is unique, while the solution $w_{h}^{n}$ is unique on $\Omega_{m}\left(c_{h}^{n-1}\right)$, for $m=1, \ldots, M$ and $n=1, \ldots, N$. 
Proof. Recalling the definition (75) and noting the convexity of $\psi_{1, \epsilon}(\cdot)$, we can introduce a regularized lower semicontinuous convex energy functional defined as

$$
F_{1, \epsilon}\left[c_{h, \epsilon}^{n}\right]=\int_{\Omega}\left\{\frac{\gamma}{2}\left|\nabla c_{h, \epsilon}^{n}\right|^{2}+\psi_{1, \epsilon}\left(c_{h, \epsilon}^{n}\right)+\chi_{\mathbb{R}^{+}}\left(c_{h, \epsilon}^{n}\right)\right\} d x,
$$

and rewrite system (84), analogously to (76), as

$$
\left\{\begin{array}{l}
\left(\frac{c_{h, \epsilon}^{n}-c_{h}^{n-1}}{\Delta t}, \chi\right)^{h}+\left(b\left(c_{h}^{n-1}\right) \nabla w_{h, \epsilon}^{n}, \nabla \chi\right)=0, \\
\left(w_{h, \epsilon}^{n}-\bar{\psi}_{2}^{\prime}\left(c_{h}^{n-1}\right), \phi-c_{h, \epsilon}^{n}\right)^{h}+F_{1, \epsilon}\left[c_{h, \epsilon}^{n}\right] \leqslant F_{1, \epsilon}[\phi],
\end{array}\right.
$$

for all $\chi, \phi \in S^{h}$. We can now pass to the limit in (100), considering the convergence properties (89) and (90) of Lemma 6. For any $(\chi, \phi) \in S^{h} \times S^{h}$, we have

$$
\begin{gathered}
\lim _{\epsilon \rightarrow 0}\left(\frac{c_{h, \epsilon}^{n}-c_{h}^{n-1}}{\Delta t}, \chi\right)^{h}=\left(\frac{c_{h}^{n}-c_{h}^{n-1}}{\Delta t}, \chi\right)^{h} ; \\
\lim _{\epsilon \rightarrow 0}\left(b\left(c_{h}^{n-1}\right) \nabla w_{h, \epsilon}^{n}, \nabla \chi\right)=\left(b\left(c_{h}^{n-1}\right) \nabla w_{h}^{n}, \nabla \chi\right) ; \\
\lim _{\epsilon \rightarrow 0}\left(w_{h, \epsilon}^{n}-\bar{\psi}_{2}^{\prime}\left(c_{h}^{n-1}\right), \phi-c_{h, \epsilon}^{n}\right)^{h} \geqslant\left(w_{h}^{n}-\psi_{2}^{\prime}\left(c_{h}^{n-1}\right), \phi-c_{h}^{n}\right)^{h},
\end{gathered}
$$

To derive the last limit (103), we use the convergence properties (89) and (90) of Lemma 6, the relation (77) written for the regularized discrete solutions, and the monotonicity of the operators in $\partial F_{1, \epsilon}(\cdot)$. At first, we take $\phi=2 c_{h, \epsilon}^{n}-c_{h}^{n}$ in the second equation of (100). Hence, we obtain

$$
\left(w_{h, \epsilon}^{n}-\bar{\psi}_{2}^{\prime}\left(c_{h}^{n-1}\right), c_{h, \epsilon}^{n}-c_{h}^{n}\right)^{h} \leqslant F_{1, \epsilon}\left[2 c_{h, \epsilon}^{n}-c_{h}^{n}\right]-F_{1, \epsilon}\left[c_{h, \epsilon}^{n}\right] .
$$

Considering the convergence property (89) and taking the limit for $\epsilon \rightarrow 0$ in the previous inequality, we get

$$
\limsup _{\epsilon \rightarrow 0}\left(w_{h, \epsilon}^{n}, c_{h, \epsilon}^{n}-c_{h}^{n}\right)^{h} \leqslant 0
$$

From (86) and the convergence properties (89) and (90) of Lemma 6, we note that we can rewrite $w_{h, \epsilon}^{n}$ as

$$
w_{h, \epsilon}^{n} \equiv-\hat{\mathcal{C}}_{c_{h}^{n-1}}^{h}\left[\frac{c_{h, \epsilon}^{n}-c_{h}^{n-1}}{\Delta t}\right]+\sum_{j \in J_{0}\left(c_{h}^{n-1}\right)} \mu_{j, \epsilon}^{n} \chi_{j}+\sum_{m=1}^{M} \lambda_{m, \epsilon}^{n} \Sigma_{m}\left(c_{h}^{n-1}\right)
$$

and the limit point $w_{h}^{n}$ as

$$
w_{h}^{n} \equiv-\hat{\mathcal{C}}_{c_{h}^{n-1}}^{h}\left[\frac{c_{h}^{n}-c_{h}^{n-1}}{\Delta t}\right]+\sum_{j \in J_{0}\left(c_{h}^{n-1}\right)} \alpha_{j}^{n} \chi_{j}+\sum_{m=1}^{M} \lambda_{m}^{n} \Sigma_{m}\left(c_{h}^{n-1}\right)
$$

Analogously to (77), we can write

$$
w_{h, \epsilon}^{n}-\psi_{2}^{\prime}\left(c_{h}^{n-1}\right) \in \partial F_{1, \epsilon}\left[c_{h, \epsilon}^{n}\right],
$$

which define a monotone map $c_{h, \epsilon}^{n} \rightarrow w_{h, \epsilon}^{n}-\psi_{2}^{\prime}\left(c_{h}^{n-1}\right)$ from $S^{h}$ to $S^{h}$. Let us introduce the quantity $f_{\lambda}=(1+\lambda) c_{h}^{n}-\lambda \phi$, with $\lambda \in \mathbb{R}, 0<\lambda<1$ and $\phi \in S^{h}$ with $\phi\left(x_{j}\right)=0$ if $c_{h}^{n}\left(x_{j}\right)=0$. It is clear that there exists a $\bar{\lambda}<1$ such that, for all $\lambda \leqslant \bar{\lambda}, f_{\lambda} \geqslant 0$. We moreover introduce the quantity 


$$
w_{h, \lambda}^{n} \equiv-\hat{\mathcal{C}}_{c_{h}^{n-1}}^{h}\left[\frac{f_{\lambda}-c_{h}^{n-1}}{\Delta t}\right]+\sum_{j \in J_{0}\left(c_{h}^{n-1}\right)} \alpha_{j, \lambda}^{n} \chi_{j}+\sum_{m=1}^{M} \lambda_{m, \lambda}^{n} \Sigma_{m}\left(c_{h}^{n-1}\right) .
$$

From the monotonicity property of the map $c_{h, \epsilon}^{n} \rightarrow w_{h, \epsilon}^{n}-\psi_{2}^{\prime}\left(c_{h}^{n-1}\right)$ and from the facts that $\phi\left(x_{j}\right)=0$ and $c_{h}^{n}\left(x_{j}\right)=0$ for $j \in J_{0}\left(c_{h}^{n-1}\right)$, it follows

$$
\left(w_{h, \epsilon}^{n}-w_{h, \lambda}^{n}, c_{h, \epsilon}^{n}-\left((1+\lambda) c_{h}^{n}-\lambda \phi\right)\right)^{h} \geqslant 0 .
$$

On the other hand, from (108), it follows

$$
\lambda\left(w_{h, \epsilon}^{n}, \phi-c_{h, \epsilon}^{n}\right)^{h} \geqslant-(1+\lambda)\left(w_{h, \epsilon}^{n}, c_{h, \epsilon}^{n}-c_{h}^{n}\right)^{h}-\left(w_{h, \lambda}^{n}, c_{h, \epsilon}^{n}-c_{h}^{n}\right)^{h}+\lambda\left(w_{h, \lambda}^{n}, \phi-c_{h}^{n}\right)^{h} .
$$

Taking now the limit for $\epsilon \rightarrow 0$ in (109), using (104), dividing by $\lambda$ and taking the limit for $\lambda \rightarrow 0$, we obtain

$$
\liminf _{\epsilon \rightarrow 0}\left(w_{h, \epsilon}^{n}-\bar{\psi}_{2}^{\prime}\left(c_{h}^{n-1}\right), \phi-c_{h, \epsilon}^{n}\right)^{h} \geqslant\left(w_{h}^{n}-\psi_{2}^{\prime}\left(c_{h}^{n-1}\right), \phi-c_{h}^{n}\right)^{h},
$$

which is (103) for all $\phi \in S^{h}$ with $\phi\left(x_{j}\right)=0$ if $c_{h}^{n}\left(x_{j}\right)=0$.

Since $\psi_{1, \epsilon}\left(c_{h, \epsilon}^{n}\right) \geqslant 0$ and since, due to the convergence property (89),

$$
\liminf _{\epsilon \rightarrow 0} \psi_{1, \epsilon}\left(c_{h, \epsilon}^{n}\right)=\psi_{1}\left(c_{h}^{n}\right)
$$

if $c_{h}^{n}<1$, from the Fatou's lemma and the semicontinuity property of the indicator function $\chi_{\mathbb{R}^{+}}(\cdot)$, we deduce that

$$
\begin{gathered}
\lim _{\epsilon \rightarrow 0} F_{1, \epsilon}\left[c_{h, \epsilon}^{n}\right] \geqslant F_{1}\left[c_{h}^{n}\right], \\
\lim _{\epsilon \rightarrow 0} F_{1, \epsilon}[\phi]=F_{1}[\phi] .
\end{gathered}
$$

Hence, the limit point $\left(c_{h}^{n}, w_{h}^{n}\right)$ satisfies, for each $(\chi, \phi) \in S^{h} \times S^{h}$, with $\phi\left(x_{j}\right)=0$ if $c_{h}^{n}\left(x_{j}\right)=0$,

$$
\left\{\begin{array}{l}
\left(\frac{c_{h}^{n}-c_{h}^{n-1}}{\Delta t}, \chi\right)^{h}+\left(b\left(c_{h}^{n-1}\right) \nabla w_{h}^{n}, \nabla \chi\right)=0, \\
\left(w_{h}^{n}-\psi_{2}^{\prime}\left(c_{h}^{n-1}\right), \phi-c_{h}^{n}\right)^{h}+F_{1}\left[c_{h}^{n}\right] \leqslant F_{1}[\phi] .
\end{array}\right.
$$

Finally, since $c_{h}^{n}<1$ (see Lemma 7) and $\psi_{1}\left(c_{h}^{n}\right)$ is convex and Lipschitz continuous for $c_{h}^{n}<1$, system (110) is equivalent to system (74) (see (76)), hence, the limit point $\left(c_{h}^{n}, w_{h}^{n}\right)$ is the unique solution of Problem $\mathrm{P}^{h}$. Recalling Remark 1, note that, if (74) is valid for each $(\chi, \phi) \in S^{h} \times K^{h}$, with $\phi\left(x_{j}\right)=0$ if $c_{h}^{n}\left(x_{j}\right)=0$, it is also valid for each $(\chi, \phi) \in S^{h} \times K^{h}$.

\section{4 | CONVERGENCE ANALYSIS}

In this section, we present the convergence analysis for the discrete scheme (74) in the case $d=1$ (see Remark 3).

We associate to the sequence of discrete solutions $c_{h}^{n}$ to Problem $\mathrm{P}^{h}$ the following continuous in time approximation:

$$
C_{h}(t):=\frac{t-t_{n-1}}{\Delta t} c_{h}^{n}+\frac{t_{n}-t}{\Delta t} c_{h}^{n-1},
$$

for $t \in\left[t_{n-1}, t_{n}\right], n=1, \ldots, N$, which is a family of linear time interpolants that depend on the parameters $h$ and $\Delta t$. We also define the piecewise constant-in-time functions

$$
\begin{aligned}
& C_{h}^{+}(t):=c_{h}^{n}, \quad C_{h}^{-}(t):=c_{h}^{n-1}, \\
& W_{h}^{+}(t):=w_{h}^{n}, \quad W_{h}^{-}(t):=w_{h}^{n-1},
\end{aligned}
$$

for $t \in\left(t_{n-1}, t_{n}\right], n=1, \ldots, N$. 
By multiplying System 74 by a $C_{0}^{\infty}([0, T])$ function and integrating in time from 0 to $T$, we obtain that $\left(C_{h}, W_{h}\right)$ satisfies the following weak formulation:

Find $\left(C_{h}, W_{h}\right) \in L^{2}\left(0, T ; K^{h}\right) \times L^{2}\left(0, T ; S^{h}\right)$ such that, for all $(\chi, \phi) \in L^{2}\left(0, T ; S^{h}\right) \times L^{2}\left(0, T ; K^{h}\right)$

$$
\left\{\begin{array}{l}
\int_{0}^{T}\left[\left(\frac{\partial C_{h}}{\partial t}, \chi\right)^{h}+\left(b\left(C_{h}^{-}\right) \nabla W_{h}^{+}, \nabla \chi\right)\right] d t \quad=0, \\
\int_{0}^{T}\left[\gamma\left(\nabla C_{h}^{+}, \nabla\left(\phi-C_{h}^{+}\right)\right)+\left(\psi_{1}^{\prime}\left(C_{h}^{+}\right), \phi-C_{h}^{+}\right)^{h}\right] d t \geqslant \int_{0}^{T}\left(W_{h}^{+}-\psi_{2}^{\prime}\left(C_{h}^{-}\right), \phi-C_{h}^{+}\right)^{h},
\end{array}\right.
$$

with $C_{h}(0)=c_{h}^{0}$.

To pass to the limit in $(113)$ as $(h, \Delta t) \rightarrow(0,0)$ and identify the system satisfied by the limit points, we need the following result, whose proof is similar to those presented in Barrett et $\mathrm{al}^{23}$ (with the only complication that we have to use Lemma 7) and therefore is not being reported here.

Lemma 8. Let $d=1$ and $c_{h}^{0}=\pi_{h}\left(c_{0}\right)$, with $0 \leqslant c_{0}<1$ and $\left|c_{0}\right|_{1} \leqslant C$. There exist a subsequence of continuous and piecewise constant in time interpolants (111) and(112), and functions $c \in L^{\infty}\left(0, T ; H^{1}(\Omega)\right) \cap H^{1}\left(0, T ;\left(H^{1}(\Omega)\right)^{\prime}\right) \cap C_{x, t}^{\frac{1}{2}, \frac{1}{8}}\left(\bar{\Omega}_{T}\right)$ and $w \in L_{l o c}^{2}(0<c<1)$ with $\frac{\partial w}{\partial x} \in L_{l o c}^{2}(0<c<1)$, such that, for $(h, \Delta t) \rightarrow(0,0)$,

$$
\begin{gathered}
C_{h}, C_{h}^{ \pm}-c \text { weakly in } L^{2}\left(0, T ; H^{1}(\Omega)\right), \\
C_{h}, C_{h}^{ \pm} \rightarrow c \text { uniformly on } \bar{\Omega}_{T}, \\
W_{h}^{+} \rightarrow w, \quad \frac{\partial W_{h}^{+}}{\partial x} \rightarrow \frac{\partial w}{\partial x} \quad \text { weakly in } L_{l o c}^{2}(0<c<1),
\end{gathered}
$$

where $\{0<q<1\}:=\left\{(x, t) \in \Omega_{T}: 0<q(x, t)<1\right\}$.

Remark 3. In the case $d=1$, the uniform convergence (115), together with the convergence result (116), allows to take the limit as $(h, \Delta t) \rightarrow(0,0)$ of the degenerate elliptic term in the first equation of system (113) on the set $\{0<c<1\}$. To the best of our knowledge, in the case $d>1$, it does not exist in the literature a convergence result, which shows the convergence of the discrete solution of (113) to the solution of a weak formulation of the continuum problem.

We can now obtain the limit equations of system $(113)$ as $(h, \Delta t) \rightarrow(0,0)$. Indeed, setting $\int_{0<c<1}() d t:,=\int_{0}^{T}(,)_{D_{0}^{+}(t)} d t$, we have the following convergence result, whose proof, being similar to that introduced in Barrett et $\mathrm{al}^{23}$ (with the only complication that we have to use Lemma 7) is not reported here.

Theorem 5. The limit point $(c, w)$ of Lemma 8 satisfies the weak formulation

$$
\left\{\begin{array}{r}
\int_{0}^{T}\left\langle\frac{\partial c}{\partial t}, \eta\right\rangle d t+\int_{0<c<1}\left(b(c) \frac{\partial w}{\partial x}, \frac{\partial \eta}{\partial x}\right) d t=0, \quad \forall \eta \in L^{2}\left(0, T ; H^{1}(\Omega)\right), \\
\int_{0<c<1} \gamma\left(\frac{\partial c}{\partial x}, \frac{\partial \theta}{\partial x}\right) d t+\int_{0<c<1}\left(\psi^{\prime}(c), \theta\right) d t-\int_{0<c<1}(w, \theta) d t=0, \\
\forall \theta \in L^{2}\left(0, T ; H^{1}(\Omega)\right),
\end{array}\right.
$$

with $c(\cdot, 0)=c_{0}(\cdot)$, and with $\operatorname{supp}(\theta) \subset\{0<c<1\}$.

\section{5 | NUMERICAL RESULTS}

After proving the existence and uniqueness and the convergence of the discrete solution, we have implemented the numerical algorithm for solving the variational inequality at each time step in Problem $\mathrm{P}^{h}$. Following the splitting procedure proposed in Barrett et al, ${ }^{23}$ we used the following iterative scheme: 
Require: $\mu>0$ (a relaxation parameter), $c_{h}^{n-1}, w_{h}^{n-1}$;

for $k \geq 0$ do

Initialization

$$
c_{h}^{n, 0}=c_{h}^{n-1}, w_{h}^{n, 0}=w_{h}^{n-1}
$$

Step 1 Find $Z^{n, k} \in S_{h}$ such that $\forall q \in S_{h}$ :

$$
\left(Z^{n, k}, q\right)^{h}=\left(c_{h}^{n, k}, q\right)^{h}-\mu\left[\lambda\left(\nabla c_{h}^{n, k}, \nabla q\right)+\left(\psi_{2}^{\prime}\left(c_{h}^{n-1}\right)-w_{h}^{n, k}, q\right)^{h}\right] ;
$$

Step 2 Find $c_{h}^{n, k+1 / 2} \in K_{h}, \forall r \geq 0$, such that:

if $j \in J_{0}\left(c_{h}^{n-1}\right)$ then

$c_{h}^{n, k+1 / 2}\left(x_{j}\right) \leftarrow c_{h}^{n-1}\left(x_{j}\right)$

else

$$
\left(c_{h}^{n, k+1 / 2}\left(x_{j}\right)+\mu \psi_{1}^{\prime}\left(c_{h}^{n, k+1 / 2}\right)\left(x_{j}\right)-Z^{n, k}\left(x_{j}\right), r-c_{h}^{n, k+1 / 2}\left(x_{j}\right)\right) \geq 0
$$

end if

Step 3 Find $\left(c_{h}^{n, k+1}, w_{h}^{n, k+1}\right) \in S_{h} \times S_{h}, \forall q \in S_{h}$, such that:

$$
\left\{\begin{array}{l}
\left(\frac{c_{h}^{n, k+1}-c_{h}^{n-1}}{\Delta t}, q\right)^{h}+\left(\nabla w_{h}^{n, k+1}, \nabla q\right)=\left(\left[1-b\left(c_{h}^{n-1}\right)\right] \nabla w_{h}^{n, k}, \nabla q\right) \\
\left(c_{h}^{n, k+1}, q\right)^{h}+\mu\left[\lambda\left(\nabla c_{h}^{n, k+1}, \nabla q\right)+\left(\psi_{2}^{\prime}\left(c_{h}^{n-1}\right)-w_{h}^{n, k+1}, q\right)^{h}=\left(2 c_{h}^{n, k+1 / 2}-Z^{n, k}, q\right)^{h} .\right.
\end{array}\right.
$$

if $\left\|c_{h}^{n, K+1}-c_{h}^{n, K}\right\|_{0, \infty}<10^{-6}$ then

$\left(c_{h}^{n}, w_{h}^{n}\right) \leftarrow\left(c_{h}^{n, K+1}, w_{h}^{n, K+1}\right) ;$ break.

end if

end for

The scalar inequality in (118) is solved using a projected gradient method, introducing an approximative analogue of the set $J_{+}\left(c_{h}^{n-1}\right)$ where $c_{h}^{n-1}>10^{-6}$ is meant for $c_{h}^{n-1}>0$. We remark that this approximation introduces a small error in the mass conservation of the algorithm. We note that, by solving the discrete problem (74) without introducing the positivity constraint and without recurring to the previous algorithm, but regularizing the degeneracy of the mobility in $c_{h}^{n-1}=0$, we could obtain negative values of $c_{h}^{n}$, which would make the discrete problem unstable. In this case, filtering out negative values of $c_{h}^{n}$ could introduce considerable errors in the mass conservation, unless we would consider very fine meshes.

To test the accuracy of the proposed numerical procedure, let us now consider the evolution of a system characterized by an initial concentration with a small uncorrelated white noise over a constant value $c_{0}$. Since we set $c_{0}<\bar{c}$, the system undergoes a spinodal decomposition and evolves, after a transitory regime, towards an equilibrium state consisting of regions, which are rich $\left(c \sim c^{*}\right)$ or empty $(c=0)$ of cells. The main features of the phase order dynamics are predicted by the classical theory of coarsening in systems with a locally conserved order parameter, described, eg, in Bray ${ }^{41}$ and Chatelain et al. ${ }^{36}$

In the following subsections, we will investigate the spinodal decomposition dynamics described by the solution of Problem $\mathrm{P}^{h}$ for different average values of initial concentration and homogeneous Neumann boundary conditions both for the $d=1$ and $d=2$ cases. For the latter case, we will also study the coarsening dynamics for long time scale solutions with the same set of average values of initial concentration as that introduced in the study of the spinodal decomposition dynamics and with periodic boundary conditions.

\section{1 | Test cases in one space dimension}

Let us first analyze the system evolution in the one-dimensional case. We consider 3 test cases in which the initial value $c_{0}$ is chosen to be a small uniformly distributed random perturbation around the values $c_{0}=0.05, c_{0}=c^{*} / 2=0.3$, and $c_{0}=0.36$. We consider homogeneous Neumann boundary conditions as in (6). We set $\gamma=0.000196, c^{*}=0.6$, and $\Delta t=10 \gamma$. The relaxation parameter is chosen to be $\mu=1 / 64$. The domain is $\Omega=(0,1)$, and a uniform partition with mesh points $x_{j}=(j-1) h, j=1, \ldots, 65$, with $h=1 / 64$, is introduced. The results are collected in Figure 3 , showing that the system exhibits 2 kinds of subregions after a transitory regime, one empty in cells, ie, $c=0$, and the other 


$$
c_{0}=0.05
$$

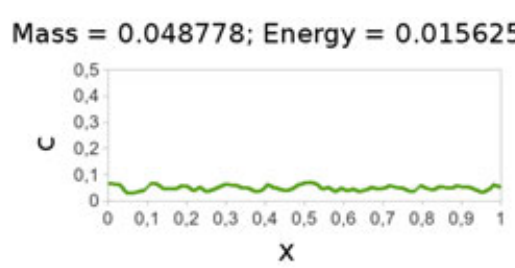

(A) $t=0$.

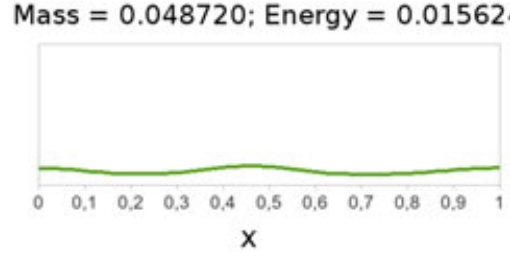

(B) $t=10.78$.

$$
c_{0}=0.3
$$

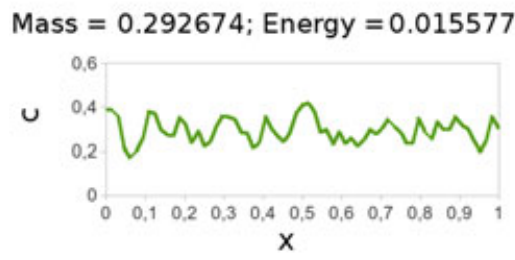

(D) $t=0$.

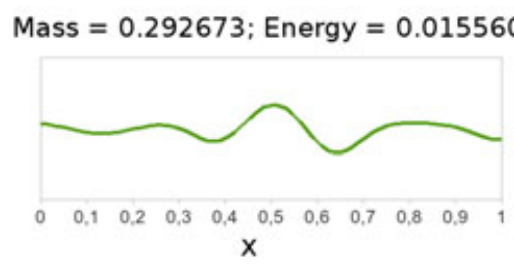

(E) $t=0.19796$.

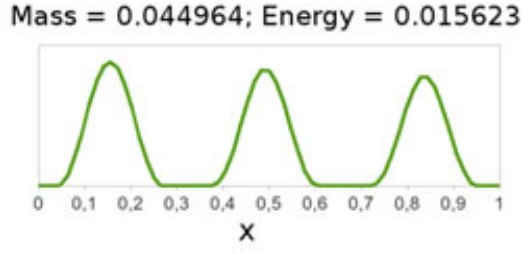

(C) $t=105.058$.

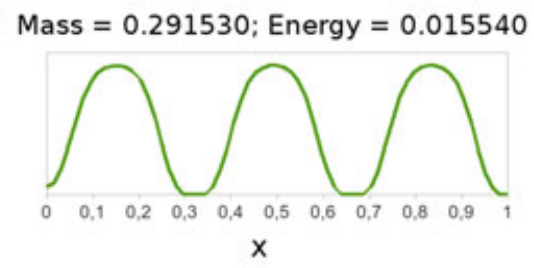

(F) $t=4.11796$.

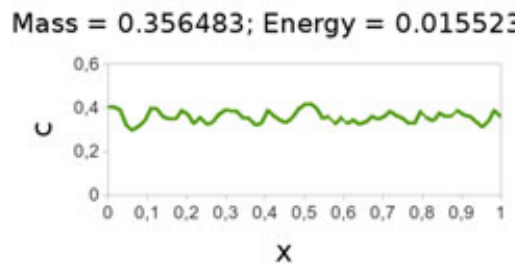

(G) $t=0$

$$
c_{0}=0.36
$$

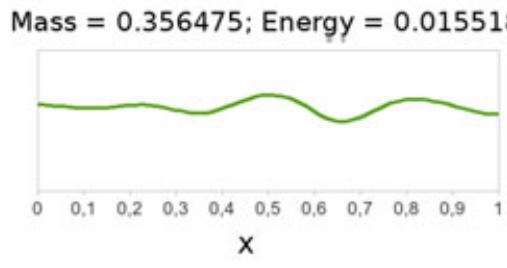

(H) $t=0.392$.

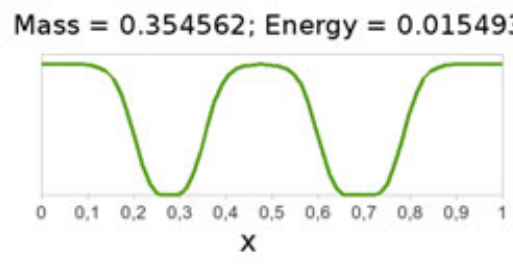

(I) $t=8.232$.

FIGURE 3 Values of $c(x)$ plotted against $x$ for $c_{0}=0.05,0.3,0.36$ at different instants of time. The values of mass and energy are reported. The values of the parameters are $\gamma=0.000196, c^{*}=0.6$, and $\Delta t=10 \gamma$ [Colour figure can be viewed at wileyonlinelibrary.com]

rich in cells, with $c \sim c^{*}$. The initial separation of the 2 phases is fast compared to the overall growth timescale of the segregated pattern.

If $c_{0}<c^{*} / 2$ (resp. $c>c^{*} / 2$ ), then the segregated solution is made of isolated clusters of cells (resp. voids), while if $c_{0}=c^{*} / 2$ the domain is equally spaced in subregions rich in each phase. We also check that the mass, ie, the value of $\left(c_{h}^{n}, 1\right)^{h}$, is conserved up to a small error and that the value of the energy $F$ (see Equation (2)) decreases.

\subsection{Test cases in 2 space dimensions}

Let us now study the evolution of the system in 2 space dimensions. The set of initial and boundary values and needed parameters is the same of the 1D case (except for $\mu=3 / 128$ here). The domain is $\Omega=(-3,3) \times(-3,3)$, and a uniform partition of 128-by-128 triangular elements is introduced.

The results are reported in Figure 4, showing the phase separation dynamics for the case $c_{0}=0.05$. As expected, after a transitory regime, the system evolves towards the formation of circular clusters of cells. The initial transitory regime is characterized by the appearance of maze-like patterns. We also check that the mass, ie, the value of $\left(c_{h}^{n}, 1\right)^{h}$, is conserved up to a small error and that the value of the energy $F$ (see Equation 2) decreases.

In Figure 5, we compare the simulation results of the degenerate case versus the ones obtained with constant mobility obtained using a $c_{0}=0.05, c_{0}=0.3$, and $c_{0}=0.36$. We can observe that in the degenerate case there is little evolution of neighboring maze-like domains, whereas in the constant mobility case such structures grow over time. Moreover, the separation of the 2 components happens at a faster timescale for the constant mobility case.

From Figure 5, we can observe that, in the case $c_{0}=0.05$, the system tends to create isolated clusters of cells, whereas the system forms maze-like patterns and cell subdomains tend to occupy half the space in the case $c_{0}=c^{*} / 2$. Finally, the system tends to form isolated circular domains empty of cells in the case $c_{0}=0.36$. 


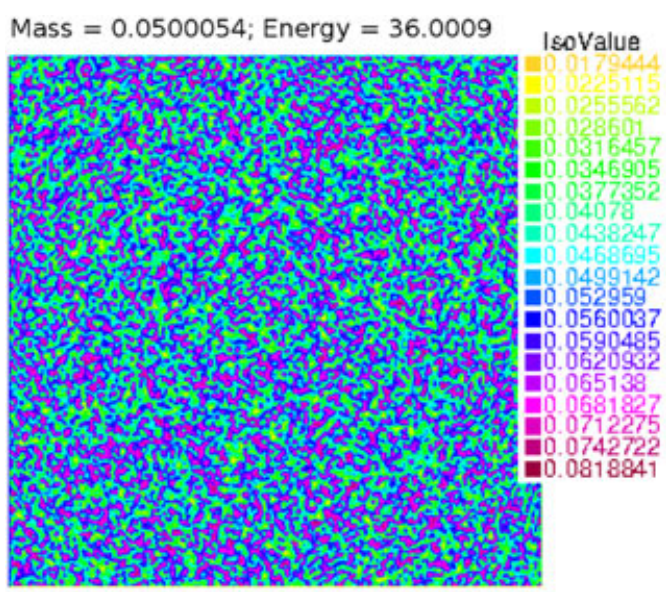

(A) $c_{0}=0.05, t=0$.

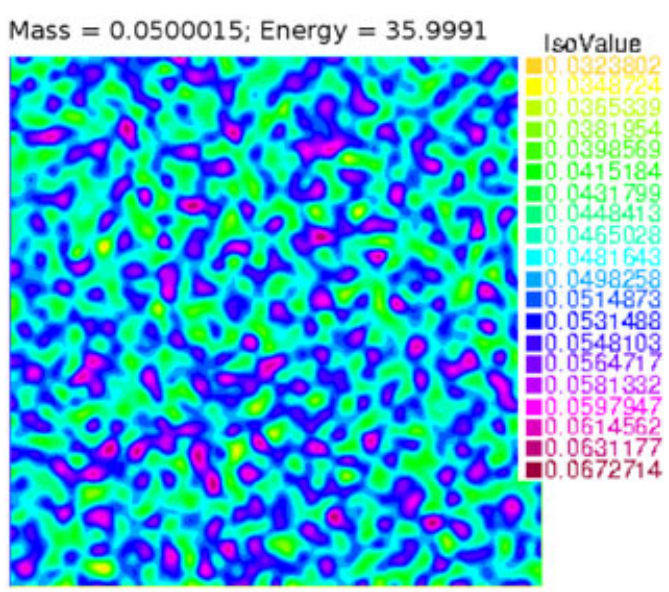

(B) $c_{0}=0.05, t=0.392$.

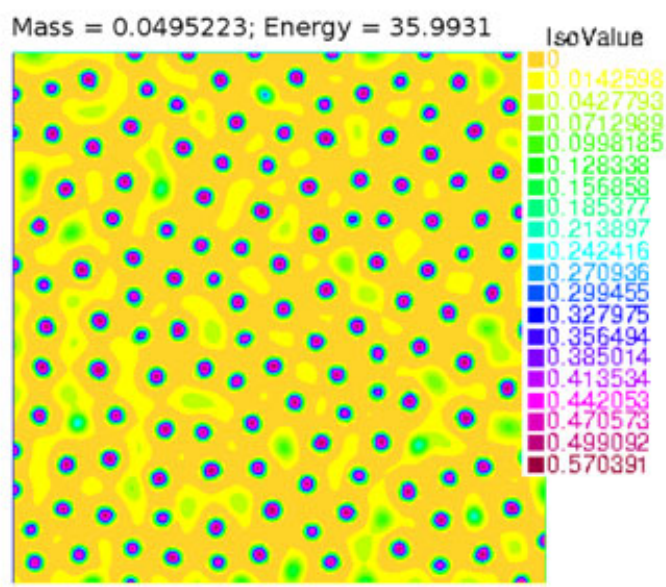

(C) $c_{0}=0.05, t=12.936$.

FIGURE 4 Values of $c(x, y)$ for $t=0 \mathrm{~s}, t=0.392 \mathrm{~s}$, and $t=9.80196 \mathrm{~s}$. The values of mass and energy are reported. The values of the parameters are $\gamma=0.000196, c^{*}=0.6$, and $\Delta t=10 \gamma$ [Colour figure can be viewed at wileyonlinelibrary.com]

\section{3 | Phase-ordering dynamics in 2 space dimensions}

The coarsening domains in CH-type models are characterized by a unique time-dependent length scale $L(t)$. For systems with a conserved order parameter and constant mobility (like the classical $\mathrm{CH}$ equation), the characteristic domain size obeys the Lifshitz-Slyozow (LS) growth law $L(t) \sim t^{1 / 3}$, (see, eg, Bray ${ }^{41}$ and Puri et $\mathrm{al}^{42}$ ). The evolution of a single phase subdomain with typical length scale $L_{i}$ at time $t \geqslant 0$ can follow 2 possible paths, as described, eg, in Bray ${ }^{41}$ : either they can shrink by diffusion if $L_{i}<L(t)$ or grow by absorbing material from the other phase if $L_{i}>L(t)$. In the standard $\mathrm{CH}$ equation with degenerate mobility, 2 limiting behaviors are typically encountered. If the degeneration set, consisting of pure phases, coincides with the set of stable equilibrium points of the double-well potential, then Mazenko's technique predicts a growth law $L(t) \sim t^{1 / 4}$, since the surface diffusion mechanism dominates, as described in Puri et al. ${ }^{42}$ Upper bounds on coarsening rates, obtained by interpolation inequalities and energy estimates, which enforce the $1 / 3$ and $1 / 4$ growth laws for the constant and the degenerate mobility cases respectively are obtained in Kohn and Otto ${ }^{43}$ and Novick-Cohen and Shishkov. ${ }^{44}$ Conversely, if the degeneration occurs for the unstable equilibrium point $c=0$, then the LS growth law is recovered, and bulk diffusion dominates, as obtained in Chatelain et al. ${ }^{36}$ For what concerns the case of the degenerate $\mathrm{CH}$ equation with a single-well potential (9), a growth law $L(t) \sim t^{0.37}$ is obtained in Chatelain et al, ${ }^{36}$ which is similar to the LS growth law associated to the standard $\mathrm{CH}$ equation with constant mobility. This might be associated to the fact that the stable equilibrium point $c=c^{*}$ of (9) is not a pure phase on which the mobility (10) vanishes, and the pure phase $c=0$ on which the mobility degenerate is an unstable equilibrium point of (9): The growth driven by bulk diffusion competes with the surface diffusion mechanism. Following Bray, ${ }^{41}$ it is found that the structure factor exhibits a dynamical scaling, 


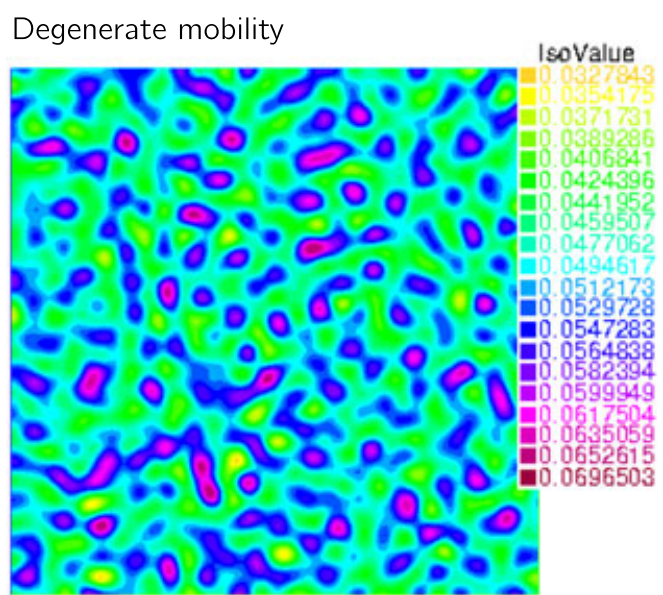

(A) $c_{0}=0.05, t=1.96196$.

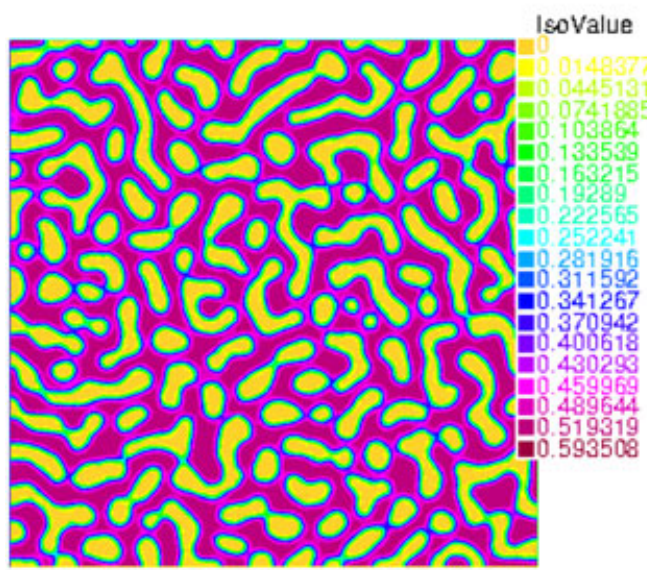

(C) $c_{0}=0.3, t=1.96196$.

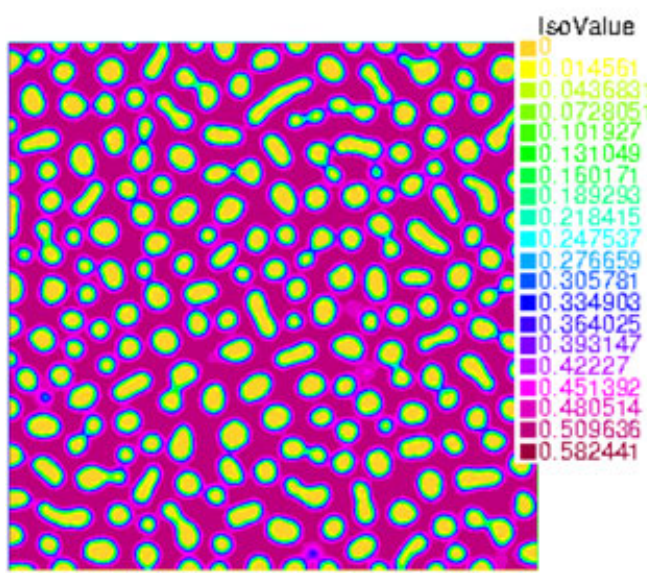

(E) $c_{0}=0.36, t=1.96196$.

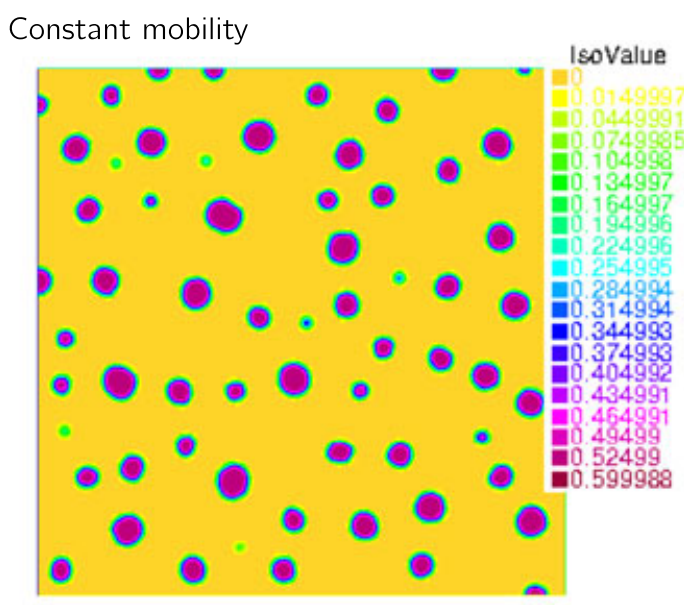

(B) $c_{0}=0.05, t=1.96196$.

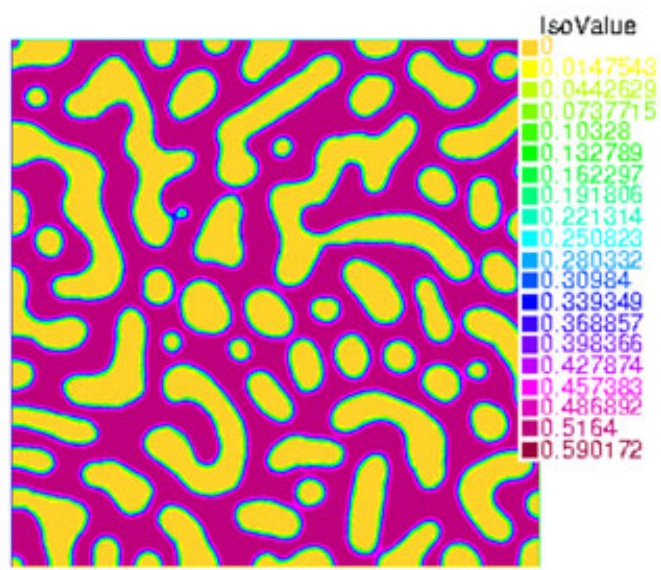

(D) $c_{0}=0.3, t=1.96196$.

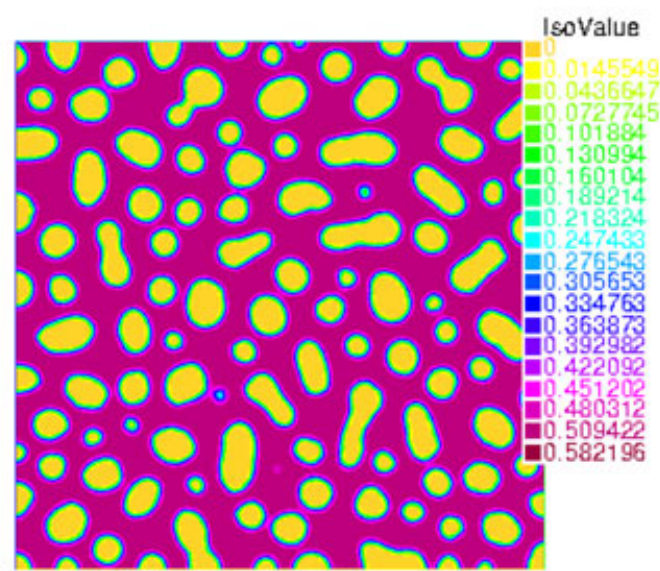

(F) $c_{0}=0.36, t=1.96196$.

FIGURE 5 Values of $c(x, y)$ for $c_{0}=0.05, c_{0}=0.3$, and $c_{0}=0.36$ for different instant of times during spinodal decomposition, for the degenerate (left panels) and constant mobility (right panels) cases. The values of the parameters are $\gamma=0.000196, c^{*}=0.6$, and $\Delta t=10 \gamma$ [Colour figure can be viewed at wileyonlinelibrary.com]

$$
S(k, t)=L(t)^{d} \mathcal{F}(k L(t)),
$$

where $S(k, t)$ is the spherically averaged time-dependent structure factor, ie, the average on the angles of the wave vector of the Fourier transform of the equal time correlation function of the solution, $d$ is the space dimension and $\mathcal{F}(\cdot)$ is a time-independent master function. A definition of the typical length scale of the system at time $t$ is given by the inverse of the first moment of the spherically averaged structure factor, $L(t)=<k>^{-1}$, with 

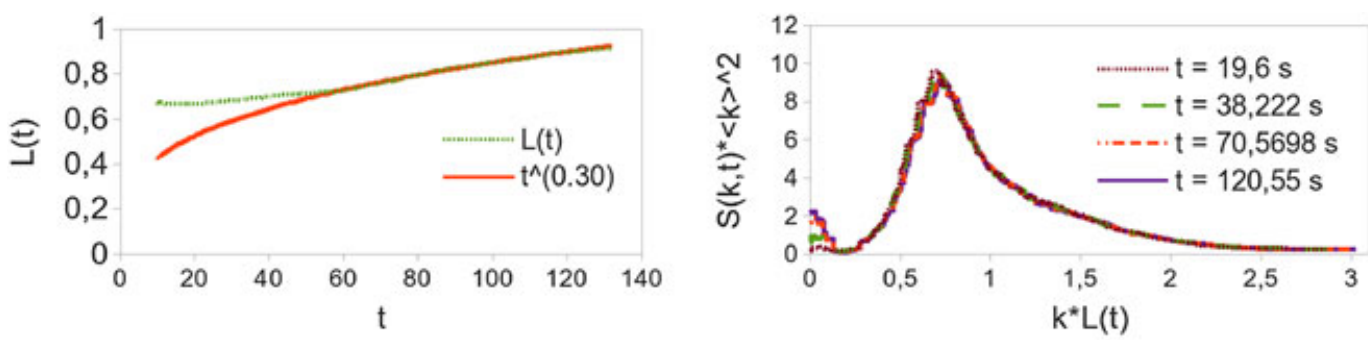

(A) $c_{0}=0.05, L(t) \sim t^{0.3}$.

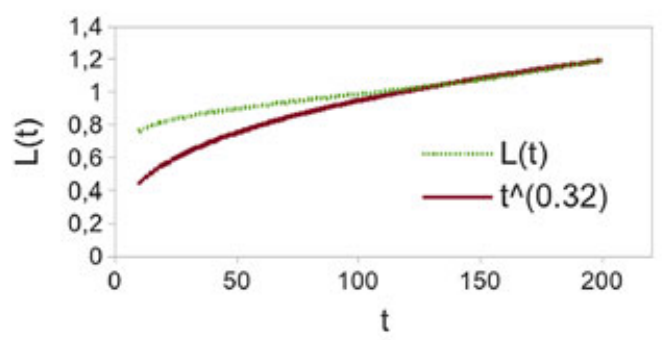

(B) $c_{0}=0.05$

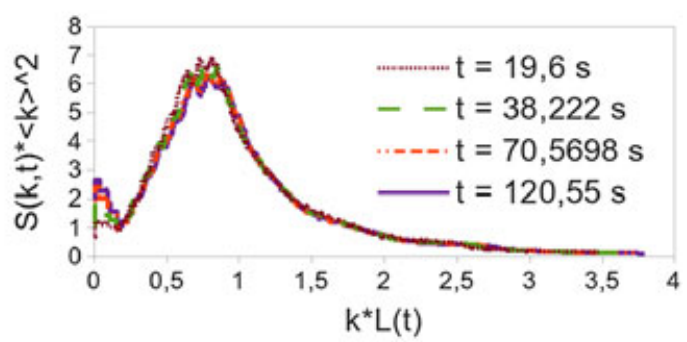

(C) $c_{0}=0.3, L(t) \sim t^{0.32}$.

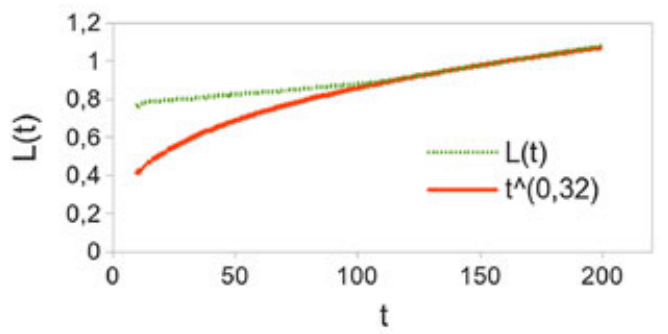

(E) $c_{0}=0.36, L(t) \sim t^{0.32}$.

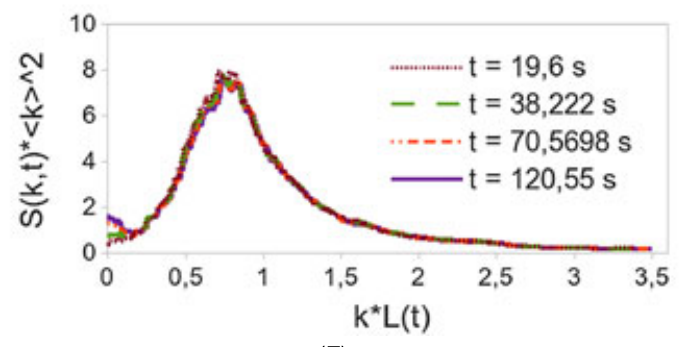

(F) $c_{0}=0.36$.

FIGURE 6 Left panels: $L(t)$ in function of $t$ superposed to a power growth law $t^{\alpha}$ for the late stages of time evolution, where the exponent $\alpha$ is obtained by a linear regression analysis, with $\alpha=0.3$ in the case $c_{0}=0.05$, and $\alpha=0.32$ in the cases $c_{0}=0.3$ and $c_{0}=0.36$. Right panels: spherically averaged scaled structured function $S(k, t)<k>^{2}$ in function of $k L(t)$ for the late time solutions of the systems evolved from the initial conditions $c_{0}=0.05, c_{0}=0.3$, and $c_{0}=0.36$. [Colour figure can be viewed at wileyonlinelibrary.com]

$$
<k=\frac{\int d k k S(k, t)}{\int d k S(k, t)} .
$$

We study 3 test cases with the same initial data $c_{0}$ as in the previous test cases and with periodic boundary conditions. In Figure 6, setting $d=2$ in (119), we plot the length scale $L(t):=<k>^{-1}$ in function of time and the spherically averaged scaled structured function $S(k, t)<k>^{2}$ in function of $k L(t)$ for the late time solutions of the systems evolved from the initial conditions $c_{0}=0.05, c_{0}=0.3$, and $c_{0}=0.36$.

The spherically averaged not normalized time-dependent structure factor $s(k, t)$ is calculated, following Mata et al, ${ }^{45}$ as the average over all wavevectors of magnitude $(k-\Delta k)$ and $(k+\Delta k)$ of the structure factor $S(\mathbf{k}, t)$, ie,

$$
s(k, t)=\frac{\sum_{k-\Delta k<|\mathbf{k}| \leqslant k-\Delta k} S(\mathbf{k}, t)}{\sum_{k-\Delta k<|\mathbf{k}| \leqslant k-\Delta k} 1},
$$

which

$$
S(\mathbf{k}, t)=\left\langle\frac{1}{N}\left|\sum_{\mathbf{r}} e^{-i \mathbf{k} \cdot \mathbf{r}}[c(\mathbf{r}, t)-<c>]\right|^{2}\right\rangle,
$$

where, in (121), the sum runs over the lattice points positions, $N=L^{2}$ is the total number of points in the lattice, $L$ is the linear size of the lattice, $\langle c\rangle$ is the spatial average of $c$ over the lattice and the outer braces $\langle\cdot\rangle$ stand for ensemble averaging. The summation in $\mathbf{r}$ in Equation 121 is calculated as a Fourier discrete transform, with $\mathbf{k}=2 \pi \mathbf{n} / L$, where the vector $\mathbf{n}=\left(n_{1}, n_{2}\right), n_{1}, n_{2}=0, \ldots, \sqrt{N}-1$, indicates the positions in the dual lattice. We set $\Delta k=(2 \pi / L) l$, with $l$ a real value near one for which the plot of the structure function is smooth. For $n_{1}, n_{2}>L / 2$, we reassign $n_{1}=\left(L-n_{1}-1\right), n_{2}=$ 
$\left(L-n_{2}-1\right)$. The normalized spherically averaged time-dependent structure factor $S(k, t)$ in (119) is then

$$
S(k, t)=\frac{s(k, t)}{\left\langle c^{2}>-<c\right\rangle^{2}} .
$$

The length scale $L(t)$ is calculated as $L(t)=1 / k_{1}(t)$, where

$$
k_{1}(t)=\frac{\sum_{k} k s(k, t)}{\sum_{k} s(k, t)}
$$

is the first moment of $s(k, t)$.

We observe that the length scale of the systems exhibits a power law evolution $L(t) \sim t^{\alpha}$ at the late stages of evolution with $\alpha=0.30$ for $c_{0}=0.05$ and $\alpha=0.32$ for $c_{0}=0.3$ and $c_{0}=0.36$, close to the LS law. We note that the best coefficient $\alpha$ is calculated using the ordinary least squares method in a linear regression analysis of the set of data $\{\log (t), \log (L(t))\}$, starting from a value of the parameter $t$ from which $L(t)$ starts to show a power growth law after an initial plateau. Moreover, we recover the classical result that the structure factors collapse on a time-independent master function, showing the self-similar scaling behavior (119) for standard phase-ordering dynamics.

\section{6 | CONCLUSIONS}

In this work, we have considered a $\mathrm{CH}$-type equation with degenerate mobility and single-well potential. In contrast to the model studied in the literature, where the degeneracy and the singularity sets coincide, here, we deal with a degeneracy $\{c=0, c=1\}$ and a singularity $\{c=1\}$. This constitutive choice introduces further complications, since $\{c=0\}$ is an unstable equilibrium point, and the singularity in $c=1$ does not guarantee that $c \geqslant 0$.

In Section 2, we have studied the existence of different classes of weak solutions of the problem and their positivity properties for the cases of spatial dimension $d=1$ and $d=2,3$ separately.

In Section 3, we have formulated an FEM approximation with continuous finite elements where we have enforced the positivity of the solution by means of a discrete variational inequality. We have proved the existence and uniqueness of the discrete solution, using a regularization approach. Moreover, we have generalized the earlier results of Barrett et $\mathrm{al}^{23}$ using some properties of subdifferential calculus for avoiding the introduction of an acute partitioning of the domain.

In Section 4, we have established the convergence in one space dimension.

In Section 5, we have presented the numerical algorithm used for solving the discrete variational inequality and we have performed simulations both in 1 and 2 space dimensions. We find that the dynamics of the spinodal decomposition for the solution of Problem $\mathrm{P}^{h}$ is, to a certain extent, analogous to the one obtained in standard phase-ordering dynamics. In fact, the geometry of the segregated domains is driven by the initial value of the concentration, with the appearance of isolated clusters of cells for $c_{0}<c^{*} / 2$ (see Figure 4) and a maze-like pattern for $c_{0}=c^{*} / 2$, see Figure 5 . A different feature of this model concerns the growth and scaling laws of phase ordering. While the degenerate $\mathrm{CH}$ equation with double-well potential is dominated by a surface diffusion mechanism at long timescales, our model follows a Lifshitz-Slyozow growth law for the characteristic length scale of the emerging patterns. Similar to the classical $\mathrm{CH}$ with constant mobility, this asymptotic behavior highlights the dominance of growth by bulk diffusion. These results on the phase-ordering dynamics are finally collected in Figure 6, also showing the existence of a master curve for the structure function.

A further development of this work will concern the error analysis of the discrete solution, which will be presented in a forthcoming paper.

Future work will be focused on the analysis of this model using a finite element approximations with discontinuous elements.

\section{ACKNOWLEDGEMENTS}

Paola F. Antonietti has been partially supported by SIR Project no. RBSI14VT0S PolyPDEs "Non-conforming polyhedral finite element methods for the approximation of partial differential equations" funded by MIUR. Paola F. Antonietti is also a member of GNCS of the Istituto Nazionale di Alta Matematica (INdAM). Pasquale Ciarletta has been partially supported by the AIRC MFAG grant 17412 and by the Start-up Packages and PhD Program project, co-funded by Regione Lombardia through the Fondo per lo sviluppo e la coesione 20072013-formerly FAS. He is a member of GNFM of the Istituto Nazionale di Alta Matematica (INdAM). Maurizio Grasselli is a member of GNAMPA of the Istituto Nazionale 
di Alta Matematica (INdAM). Marco Verani has been partially supported by GNCS-INDAM and by the Italian research grant PRIN 2012 2012HBLYE4 “Metodologie innovative nella modellistica differenziale numerica."

\section{ORCID}

Abramo Agosti $($ http://orcid.org/0000-0001-5706-3772

\section{REFERENCES}

1. Cahn JW, Hilliard JE. Free energy of a nonuniform system. I. Interfacial free energy. J Chem Phys. 1958;28(2):258-267.

2. Cahn JW. On spinodal decomposition. Acta Metall. 1961;9(9):795-801.

3. Lee D, Huh JY, Jeong D, Shin J, Yun A, Kim J. Physical, mathematical, and numerical derivations of the Cahn-Hilliard equation. Comput Mater Sci. 2014;81:216-225.

4. Novick-Cohen A. The Cahn-Hilliard Equation. Handbook of Differential Equations: Evolutionary Equations. Vol. IV, 201228, Handb. Differ. Equ. Elsevier/North-Holland: Amsterdam, 2008.

5. Elliott CM, Zheng S. On the Cahn-Hilliard equation. Arch Ration Mech Anal. 1986;96(4):339-357.

6. Cherfils L, Miranville A, Zelik S. The Cahn-Hilliard equation with logarithmic potentials. Milan J Math. 2011;79(2):561-596.

7. Elliott CM, Garcke H. On the Cahn-Hilliard equation with degenerate mobility. SIAM J Math Anal. 1996;27(2):404-423.

8. Cahn JW, Elliott CM, Novick-Cohen A. The Cahn-Hilliard equation with a concentration dependent mobility: motion by minus the laplacian of the mean curvature. European J Appl Math. 1996;7(03):287-301.

9. Schimperna G, Zelik S. Existence of solutions and separation from singularities for a class of fourth order degenerate parabolic equations. T Am Math Soc. 2013;365(7):3799-3829.

10. Allen SM, Cahn JW. Ground state structures in ordered binary alloys with second neighbor interactions. Acta Metall. 1972;20(3):423-433.

11. McMaster LP. Aspects of liquid-liquid phase transition phenomena in multicomponent polymeric systems. Adv Chem Ser. 1975;142:43.

12. Zeng Y, Bazant MZ. Phase separation dynamics in isotropic ion-interaction particles. SIAM J Appl Math. 2014;74(4):980-1004.

13. Erlebacher J, Aziz MJ, Karma A, Dimitrov N, Sieradzki K. Evolution of nanoporosity in dealloying. Nat. 2001;410(6827):450-453.

14. Bertozzi AL, Esedoglu S, Gillette A. Inpainting of binary images using the Cahn-Hilliard equation. IEEE Trans Image Process. 2007;16(1):285-291.

15. Tremaine S. On the origin of irregular structure in Saturn's rings. Astron J. 2003;125:894-901.

16. Gurtin ME. Generalized Ginzburg-Landau and Cahn-Hilliard equations based on a microforce balance. Phys D. 1996;92(3):178-192.

17. Gurtin ME, Polignone D, Vinals J. Two-phase binary fluids and immiscible fluids described by an order parameter. Math Models Methods Appl Sci. 1996;6(6):815-831.

18. Lowengrub J, Truskinovsky L. Quasi-incompressible Cahn-Hilliard fluids and topological transitions. In Proceedings of the Royal Society of London. Series A, Mathematical Physical and Engineering Sciences, Ther Royal Society publishing, London, 1998;454:2617-2654.

19. Khain E, Sander LM. Generalized Cahn-Hilliard equation for biological applications. Phys Rev E. 2008;77:051129.

20. Liu QX, Doelman A, Rottschäfer V, et al. Phase separation explains a new class of self-organized spatial patterns in ecological systems. Proc Natl Acad Sci USA. 2013;110(29):11905-11910.

21. Wise SM., Lowengrub JS, Frieboes HB, Cristini V. Three-dimensional multispecies nonlinear tumor growth I: model and numerical method. J Theoret Biol. 2008;253(3):524-543.

22. Grün G. Degenerate parabolic differential equations of fourth order and a plasticity model with non-local hardening. $Z$ Anal Anwend. 1995;14(3):541-574.

23. Barrett JW, Blowey JF, Garcke H. Finite element approximation of the Cahn-Hilliard equation with degenerate mobility. SIAM J Numer Anal. 1999;37(1):286-318.

24. Grün G, Rumpf M. Nonnegativity preserving convergent schemes for the thin film equation. Numer Math. 2000;87:113-152.

25. Copetti MIM, Elliott CM. Numerical analysis of the Cahn-Hilliard equation with a logarithmic free energy. Numer Math. 1992;63(1):39-65.

26. Gómez H, Calo VM, Bazilevs Y, Hughes TJR. Isogeometric analysis of the Cahn-Hilliard phase-field model. Comput Methods Appl Mech Engrg. 2008;197(49-50):4333-4352.

27. Liu J, Dedè L, Evans JA, Borden MJ, Hughes TJR. Isogeometric analysis of the advective Cahn-Hilliard equation: spinodal decomposition under shear flow. J Comput Phys. 2013;242:321-350.

28. Kay D, Styles V, Süli E. Discontinuous Galerkin finite element approximation of the Cahn-Hilliard equation with convection. SIAM J Numer Anal. 2009;47(4):2660-2685.

29. Wells GN, Kuhl E, Garikipati KA. A discontinuous Galerkin method for the Cahn-Hilliard equation. J Comput Phys. 2006;218(2):860-877.

30. Xia Y, Xu Y, Shu CW. Local discontinuous Galerkin methods for the Cahn-Hilliard type equations. J Comput Phys. 2007;227(1):472-491.

31. Antonietti PF, Beirao da Veiga L, Scacchi S, Verani M. A $c^{1}$ virtual element method for the Cahn-Hilliard equation with polygonal meshes. SIAM J Numer Anal. 2016;54(1):34-56.

32. Chave F, Di Pietro DA, Marche F, Pigeonneau F. A Hybrid High-Order Method for the Cahn-Hilliard problem in Mixed form. SIAM J Numer Anal. 2016;54(3):1873-1898.

33. Byrne H, Preziosi L. Modelling solid tumour growth using the theory of mixtures. Math Med Biol. 2003;20(4):341-366. 
34. Chatelain C, Ciarletta P, Amar MB. Morphological changes in early melanoma development: influence of nutrients, growth inhibitors and cell-adhesion mechanisms. J Theoret Biol. 2011;290:46-59.

35. Colombo MC, Giverso C, Faggiano E, Boffano C, Acerbi F, Ciarletta P. Towards the personalized treatment of glioblastoma: integrating patient-specific clinical data in a continuous mechanical model. PLoS One. 2015;10. http://journals.plos.org/plosone/article?id=10.1371/ journal.pone. 0132887

36. Chatelain C, Balois T, Ciarletta P, Ben Amar M. Emergence of microstructural patterns in skin cancer: a phase separation analysis in a binary mixture. New J Phys. 2011;115013(13):21.

37. Adams RA. Sobolev Spaces. Academic press: New York, 1975.

38. Bernis F, Friedman A. Higher order nonlinear degenerate parabolic equations. J Differ Equ. 1990;83:179-206.

39. Kenmochi N, Niezgódka M, Pawlow I. Subdifferential operator approach to the Cahn-Hilliard equation with constraint. $J$ Differ Equ. 1995;117:320-354.

40. Ekeland I, Temam R. Convex Analysis and Variational Problems. SIAM, 1999.

41. Bray AJ. Theory of phase-ordering kinetics. Adv Phys. 1994;43(3):357-459.

42. Puri S, Bray AJ, Lebowitz JL. Phase separation kinetics in a model with order-parameter dependent mobility. Phys Rev E. 1997;56:758.

43. Kohn VR, Otto F. Upper bounds on coarsening rates. Comm Math Phys. 2002;229:375-395.

44. Novick-Cohen A, Shishkov A. Upper bounds for coarsening for the degenerate Cahn-Hilliard equation. Discrete Contin Dyn Syst. 2009;25(1):251-272.

45. Mata M, Garcia-Cervera CJ, Ceniceros HD. Ordering kinetics of a conserved binary mixture with a nematic liquid crystal component. J Nonnewton Fluid Mech. 2014;212:18-27. 